IMA Journal of Mathematical Control and Information (2016) 33, 701-722

doi:10.1093/imamci/dnv004

Advance Access publication on February 13, 2015

\title{
Relative controllability properties
}

\author{
Fritz Colonius and RalPh LetTAU* \\ Institut für Mathematik, Universität Augsburg, 86159 Augsburg, Germany \\ *Corresponding author: ralph.lettau@math.uni-augsburg.de
}

[Received on 12 August 2014; revised on 9 December 2014; accepted on 10 January 2015]

\begin{abstract}
For non-linear systems described by ordinary differential equations, the paper studies $W$-control sets which are defined as maximal subsets of complete approximate controllability within a safe region or world $W$ in the state space. In particular, their relative invariance properties and their behaviour under parameter variations are characterized. An application to invariance entropy shows that the information needed to keep a system in a subset of the state space is determined by the relatively invariant $W$-control sets.
\end{abstract}

Keywords: control sets, safe regions, invariance entropy.

\section{Introduction}

The purpose of this paper is to analyse controllability properties of non-linear systems described by ordinary differential equations under the additional requirement that a prescribed safe region $W$ (also called world in the following) in the state space $M$ is not left. The safe region corresponds to the requirement that the system should satisfy certain constraints in order to ensure integrity of the system. Thus, if a trajectory leaves the safe region $W$, then the system stops. Another interpretation is that the complement $H:=M \backslash W$ of the world $W$ is a hole in the state space, through which the system may disappear (in contrast, we think of $W$ as an island). In the theory of (uncontrolled) dynamical systems, one also speaks of 'open dynamical systems' and there is a considerable body of literature on them, cf. Demers \& Young (2006) for a survey. The analysis and computation of safe regions in control systems also originates from application areas. For example, Tomlin (1998) discusses safe regions motivated by aircraft traffic control problems.

A central notion for this paper are control sets relative to $W$, i.e. maximal subsets of complete approximate controllability within $W$. For the theory of control sets in $M$, we refer the reader to Colonius \& Kliemann (2000). Control sets and their relations to flows and semiflows have also been analysed by San Martin and coworkers in the context of semigroups in Lie groups. Here relations between the structure of semisimple Lie groups, semigroup actions and control sets have been established, cf., e.g. Patrao \& SanMartin (2007). Parameter dependence of control sets has, in particular, been analysed by Gayer (2004) and Graf (2011).

Instead of starting with a system on $M$ and restricting it to $W$, one could also start directly with a system on $W$ allowing for finite existence intervals. We prefer to start with the system on $M$, since our main interest is in the relations between the controllability properties within $W$ and the controllability properties in $M$.

The information needed to keep a control system in a subset of the state space can be described by (feedback) invariance entropy, cf. Nair (2004) and Kawan (2013). We will show that here the relatively invariant $W$-control sets play an important role, since for 'large' sets of initial values the invariance 
entropy is determined by the relatively invariant $W$-control sets. In general, it is difficult to obtain estimates or even formulas for invariance entropy. However, for the invariance entropy of control sets, good estimates are available by the results in Kawan (2012). Hence, our result reducing the computation of invariance entropy to this situation is of interest.

The original motivation for the analysis of invariant control sets is due to the fact, that they often determine the supports of stationary measures of associated random systems; cf. Arnold \& Kliemann (1987) and Colonius (2008). We hope to show in future work, that relatively invariant control sets can be used for the analysis of transient behaviour of random systems.

The contents of this paper is as follows: Section 2 presents the setting of control systems restricted to open (not invariant) subsets $W$ of the state space and specifies relevant accessibility notions. In Section 3, $W$-control sets, i.e. maximal subsets of approximate controllability restricted to $W$, are introduced and their main properties are characterized; in particular, also their parameter dependence is studied. Section 4 concentrates on relatively invariant $W$-control sets, which cannot be left without leaving $W$. These are the $W$-control sets which are closed relative to $W$ and their existence is characterized. Furthermore, Theorem 4.12 gives conditions which ensure that, under variation of a parameter $\alpha$, an invariant control set (in $M$ ) generates a family of relatively invariant $W^{\alpha}$-control sets. Section 5 presents an application to invariance entropy given in Theorem 5.2 and Section 6 illustrates the results using a model of a continuous stirred tank reactor.

Notation: For non-empty subsets $A, B$ of a metric space $X$ with metric $d$ and points $x \in X$, we let

$$
\operatorname{dist}(x, A):=\inf _{a \in A} d(x, a) \quad \text { and } \quad d_{H}(A, B):=\max \left\{\sup _{a \in A} \operatorname{dist}(a, B), \sup _{b \in B} \operatorname{dist}(b, A)\right\} \leqslant \infty .
$$

\section{Preliminaries}

Let $M$ be a connected smooth Riemannian manifold of dimension $d$ (endowed with the distance $d$ induced by the Riemannian metric) and let $X: M \times \mathbb{R}^{m} \rightarrow T M$ be a continuous map such that $X(\cdot, u), u \in \mathbb{R}^{m}$ are smooth vector fields (here and below, smooth means $C^{\infty}$ ). Consider a control system on $M$ given by

$$
\dot{x}(t)=X(x(t), u(t)), \quad u \in \mathcal{U}
$$

with $\mathcal{U}=\left\{u: \mathbb{R} \rightarrow \mathbb{R}^{m} \mid u(t) \in U\right.$ for all $t \in \mathbb{R}$, locally integrable $\}$. Here $U$ is a non-void subset of $\mathbb{R}^{m}$. For $x \in M$ and $u \in \mathcal{U}$, the unique (maximal) local solution with $\varphi(0, x, u)=x$ is denoted by $\varphi(t, x, u)$ defined on the maximal (open) interval $I(x, u)$ containing 0 .

Fix a non-void open and connected subset $W \subset M$ with compact closure clW called the world in which the system lives. The set $W$ may also be considered as a state constraint or a safe region, which the system must not leave. Naturally, $W$ is a submanifold of $M$ with equal dimension. The dynamics restricted to $W$ are determined by the restriction of $X$

$$
\dot{x}_{W}(t)=X_{W}\left(x_{W}(t), u(t)\right), \quad u \in \mathcal{U}, \text { with } X_{W}:=X_{\mid W \times \mathbb{R}^{m}} .
$$

The (unique) solutions $\varphi_{W}(t, x, u)$ of this $W$-system coincide with the solutions of system (2.1) restricted to $W$. Using compactness of the closure of $W$, one finds that for every $x \in W$ and every $u \in \mathcal{U}$ the solutions have the following property: Either $\varphi_{W}(t, x, u)=\varphi(t, x, u)$ is defined for all $t \geqslant 0$ or $\varphi\left(\tau^{+}, x, u\right) \notin W$ 
for some $\tau^{+}>0$; analogously for negative times. Thus, the existence interval of $\varphi_{W}(t, x, u)$ has the form

$$
I_{W}(x, u)=\left(-\tau^{-}(x, u), \tau^{+}(x, u)\right)
$$

with $\tau^{ \pm}(x, u)>0$, and $\tau^{ \pm}(x, u)<\infty$ implies $\varphi\left(\tau^{ \pm}(x, u), x, u\right) \notin W$; in this case $\varphi\left(\tau^{ \pm}(x, u), x, u\right)$ is an element of the boundary $\partial W$ of $W$.

REMARK 2.1 We impose the assumption that the closure of $W$ is compact, since this considerably simplifies a number of arguments, cp. in particular Proposition 2.5. Note that the boundary $\partial W=\partial(\operatorname{cl} W)$ of the compact set $\mathrm{cl} W$ is compact.

REMARK 2.2 The set $W$ is considered in the topology induced by $M$, i.e. the open sets in $W$ are of the form $A \cap W$ for an open subset $A$ of $M$. Since $W$ is open, a set $O \subset W$ is $W$-open iff it is open in $M$ and neighbourhoods in $W$ coincide with neighbourhoods in $M$. In contrast, closed sets in $W$ may not be closed in $M$ and we denote for a set $A \subset W$ the $W$-closure by $\operatorname{cl}_{W}(A)$. Furthermore, the boundary $\partial_{W} A$ with respect to $W$ of a subset $A \subset W$ is given by

$$
\partial_{W} A:=\left(\operatorname{cl}_{W}(A)\right) \cap\left(\mathrm{cl}_{W}(W \backslash A)\right)
$$

and hence $\partial_{W} A$ may be a proper subset of the boundary $\partial A$ with respect to $M$ of $A$. Furthermore, $\partial A=\partial_{W} A \cup(\partial A \cap \partial W)$.

Next we discuss reachability properties within $W$. For later purpose, we formulate the following slightly more general definition.

Definition 2.3 Consider a control system of the form (2.1) and let $A \subset M$. The $A$-reachability set of $x \in A$ up to time $T>0$ is

$$
\mathcal{O}_{\leqslant T}^{A,+}(x):=\left\{\begin{array}{l|l}
y \in A & \begin{array}{c}
\text { there are } u \in \mathcal{U} \text { and } t \in[0, T] \cap I(x, u) \text { with } \\
y=\varphi(t, x, u) \text { and } \varphi\left(t^{\prime}, x, u\right) \in A \text { for all } t^{\prime} \in[0, t]
\end{array}
\end{array}\right\} .
$$

The $A$-controllability set of $x$ up to time $T$ is

$$
\mathcal{O}_{\leqslant T}^{A,-}(x):=\left\{\begin{array}{l|l}
y \in A & \begin{array}{c}
\text { there are } u \in \mathcal{U} \text { and } t \in[0, T] \cap I(y, u) \text { with } \\
x=\varphi(t, y, u) \text { and } \varphi\left(t^{\prime}, y, u\right) \in A \text { for all } t^{\prime} \in[0, t]
\end{array}
\end{array}\right\} .
$$

Furthermore,

$$
\mathcal{O}^{A,+}(x):=\bigcup_{T>0} \mathcal{O}_{\leqslant T}^{A,+}(x) \quad \text { and } \quad \mathcal{O}^{A,-}(x):=\bigcup_{T>0} \mathcal{O}_{\leqslant T}^{A,-}(x) .
$$

If $A=M$, we just write $\mathcal{O}^{+}(x)$, etc. Considering system (2.1) and the restricted system (2.2), one has for $x \in W$

$$
\mathcal{O}_{\leqslant T}^{W,+}(x)=\left\{y \in W \mid \text { there are } u \in \mathcal{U} \text { and } t \in[0, T] \cap I_{W}(x, u) \text { with } y=\varphi_{W}(t, x, u)\right\},
$$

and analogously for the other sets defined above.

Next we construct an auxiliary system defined on the state space $W$ using a smooth cutoff function which is positive on $W$ and vanishes on the boundary of $W$. We use the following result from Lee (2013, Lemma 2.29). 
Lemma 2.4 Let $W$ be an open set in $M$. Then there exists a smooth function $\alpha: M \rightarrow[0,1]$ with $\alpha(x)>$ 0 for all $x \in W$ and $\alpha(x)=0$ for $x \in M \backslash W$.

Next we define the following auxiliary control system. Consider system (2.1) and define with a cutoff function $\alpha$ as given by Lemma 2.4

$$
\dot{y}=\alpha(y) X(y, v), \quad v \in \mathcal{U} .
$$

Note that the vector fields $\alpha(\cdot) X(\cdot, u), u \in U$, are defined on $M$ and vanish on $M \backslash W$. The local trajectories of (2.4) with initial condition $y(0)=x_{0} \in M$ are denoted by $\psi\left(\tau, x_{0}, v\right)$ for $\tau$ in a maximal (open) existence interval around $\tau_{0}=0$.

The following discussion relates accessibility properties of the three control systems (2.1), (2.2) and (2.4). We begin with the following lemma relating trajectories of (2.2) and (2.4).

Proposition 2.5 (i) For every $x_{0} \in W$ and every control $v \in \mathcal{U}$, the solution $\psi\left(\tau, x_{0}, v\right)$ of (2.4) exists for all $\tau \in \mathbb{R}$.

(ii) Let $x_{0} \in W$. For every solution $\varphi_{W}\left(t, x_{0}, u\right), t \in I_{W}\left(x_{0}, u\right)$, corresponding to a control $u \in \mathcal{U}$ one finds a control $v \in \mathcal{U}$ such that the trajectories $\left\{\varphi_{W}\left(t, x_{0}, u\right) \mid t \in I_{W}\left(x_{0}, u\right)\right\}$ for (2.2) and $\left\{\psi\left(\tau, x_{0}, v\right) \mid \tau \in \mathbb{R}\right\}$ for (2.4) coincide. Conversely, for every solution $\psi\left(\tau, x_{0}, v\right), \tau \in \mathbb{R}$, corresponding to a control $v \in \mathcal{U}$ one finds a control $u \in \mathcal{U}$ such that the trajectories $\left\{\psi\left(\tau, x_{0}, u\right) \mid \tau \in\right.$ $\mathbb{R}\}$ for (2.4) and $\left\{\varphi_{W}\left(t, x_{0}, u\right) \mid t \in I_{W}\left(x_{0}, u\right)\right\}$ for (2.2) coincide. The same is true for the positive semi-trajectories with $t \geqslant 0$ and $\tau \geqslant 0$, respectively.

Proof. (i) For every control value $u \in U$, the right-hand side $\alpha(\cdot) X(\cdot, u)$ of $(2.4)$ is smooth and vanishes on the boundary of the compact set $\mathrm{cl} W$. Using local existence and uniqueness of solutions, no trajectory $\psi\left(\cdot, x_{0}, v\right), x_{0} \in W, v \in \mathcal{U}$, can reach in finite time the boundary of $W$. Hence, the solutions of (2.4) exist for all $\tau \in \mathbb{R}$.

(ii) Consider for $x_{0} \in W$ and $u \in \mathcal{U}$ the solution $x(t):=\varphi_{W}\left(t, x_{0}, u\right), t \in I_{W}\left(x_{0}, u\right)$, and define $\rho(t):=$ $\int_{0}^{t}\left[\alpha\left(\varphi_{W}\left(s, x_{0}, u\right)\right)\right]^{-1} \mathrm{~d} s, t \in I_{W}\left(x_{0}, u\right)$. The transformation $t \mapsto \rho(t)$ is continuously differentiable with $(\mathrm{d} / \mathrm{d} t) \rho(t)=\left[\alpha\left(\varphi_{W}\left(t, x_{0}, u\right)\right)\right]^{-1} \neq 0$. Hence, the transformation is invertible and the inverse $\rho^{-1}(\tau)$ exists with

$$
\frac{\mathrm{d}}{\mathrm{d} \tau} \rho^{-1}(\tau)=\left(\frac{\mathrm{d}}{\mathrm{d} t} \rho(\cdot)_{\mid \rho^{-1}(\tau)}\right)^{-1}=\alpha\left(\varphi_{W}\left(\rho^{-1}(\tau), x_{0}, u\right)\right) .
$$

Define $y(\tau):=x\left(\rho^{-1}(\tau)\right)=\varphi_{W}\left(\rho^{-1}(\tau), x_{0}, u\right)$. Then one computes

$$
\begin{aligned}
\frac{\mathrm{d}}{\mathrm{d} \tau} y(\tau) & =\dot{x}\left(\rho^{-1}(\tau)\right) \frac{\mathrm{d}}{\mathrm{d} \tau} \rho^{-1}(\tau) \\
& =X\left(\varphi_{W}\left(\rho^{-1}(\tau), x_{0}, u\right), u\left(\rho^{-1}(\tau)\right)\right) \alpha\left(\varphi_{W}\left(\rho^{-1}(\tau), x_{0}, u\right)\right) \\
& =X(y(\tau), v(\tau)) \alpha(y(\tau)),
\end{aligned}
$$

where $v(\tau):=u\left(\rho^{-1}(\tau)\right), \tau \in \mathbb{R}$. Thus, uniqueness of the solutions implies $y(\tau)=\psi\left(\tau, x_{0}, v\right), \tau \in \mathbb{R}$.

For the converse, consider for $x_{0} \in W$ and $v \in \mathcal{U}$ the solution $y(\tau):=\psi\left(\tau, x_{0}, v\right), \tau \in \mathbb{R}$, and define $\gamma(\tau):=\int_{0}^{\tau} \alpha\left(\psi\left(\sigma, x_{0}, v\right)\right) \mathrm{d} \sigma, \tau \in \mathbb{R}$. Then one argues as above.

Finally, the time transformations keep zero fixed and preserve the orientation, hence the assertion about the semi-trajectories follows. 
Proposition 2.5(i) shows that we may restrict the state space of system (2.4) to $W$. This will be done in the sequel.

We will use the following version of local accessibility.

Definition 2.6 A control system of the form (2.1) is locally accessible at $x \in M$, if for every neighbourhood $N$ of $x$ and every $T>0$

$$
\operatorname{int} \mathcal{O}_{\leqslant T}^{N,+}(x) \neq \varnothing \quad \text { and } \quad \operatorname{int} \mathcal{O}_{\leqslant T}^{N,-}(x) \neq \emptyset .
$$

The system is locally accessible if it is locally accessible at every point of its state space $M$.

REMARK 2.7 In the literature, different versions of local accessibility can be found: Crouch calls a system locally accessible if for each $x \in M$ and neighbourhood $N$ of $x$ the reachable set $\mathcal{O}^{N,+}(x)$ has nonvoid interior. Colonius \& Kliemann (2000, p. 48) require that for each $x \in M$ and $T>0$ the sets $\mathcal{O}_{\leqslant T}^{ \pm}(x)$ have non-void interiors. Both conditions are weaker than the definition above which is appropriate for our purposes.

Throughout the rest of this paper, we assume that system (2.1) is locally accessible at every point of $W$. Since $W$ is an open subset of $M$, this is equivalent to local accessibility of (2.2) (i.e. at every point of its state space $W$ ).

DEFINITION 2.8 A control system of the form (2.1) satisfies the Lie algebra rank condition (ARC), also called the accessibility rank condition at $x_{0} \in W$, if the rank of the Lie Algebra $\operatorname{Lie}\{X(\cdot, u), u \in U\}$ generated by the vector fields $X(\cdot, u)$ evaluated at $x_{0}$ coincides with the dimension $d$ of $M$.

Since $W$ is open, condition (ARC) holds for (2.1) at a point $x_{0}$ of $W$ iff it holds for (2.2) at $x_{0}$. Local accessibility of (2.1) (or, equivalently, of (2.2)) at $x_{0} \in W$ follows if the Lie algebra rank condition (ARC) holds at $x_{0}$; cf. Sontag (1998, Chapter 4, Theorem 9). We also note the following property.

LEMma 2.9 If system (2.1) is locally accessible at every point of $W$, then

$$
\mathcal{O}^{W,+}\left(x_{0}\right) \subset \operatorname{cl}_{W}\left[\operatorname{int} \mathcal{O}^{W,+}\left(x_{0}\right)\right] \text { for every } x_{0} \in W .
$$

Proof. Let $x \in \mathcal{O}^{W,+}\left(x_{0}\right)$. By local accessibility for every neighbourhood $N \subset W$ of $x$, there exists $y \in$ $\operatorname{int} \mathcal{O}^{N,+}(x) \subset N \cap \operatorname{int} \mathcal{O}^{W,+}\left(x_{0}\right)$. Hence, there is a sequence $\left(y_{i}\right)$ with $y_{i} \in \operatorname{int} \mathcal{O}^{W,+}(x)$ converging to $x$ and $x \in \operatorname{cl}_{W}\left[\operatorname{int} \mathcal{O}^{W,+}\left(x_{0}\right)\right]$ follows.

Next we show that local accessibility of system (2.2) is equivalent to local accessibility of system (2.4).

Proposition 2.10 System (2.4) is locally accessible iff system (2.2) is locally accessible.

Proof. Assume local accessibility of (2.2). Let $x_{0} \in W$ and fix $T>0$ and a neighbourhood $N \subset W$ of $x_{0}$. Choose $N$ small enough such that $\operatorname{cl} N \cap \partial W=\emptyset$. We claim that there is $T^{\prime}>0$ such that for all $u \in \mathcal{U}$ there is $v \in \mathcal{U}$ such that

$$
\left\{\varphi_{W}\left(t, x_{0}, u\right) \mid t \in\left[0, T^{\prime}\right] \text { and } \varphi\left(t^{\prime}, x_{0}, v\right) \in N \text { for all } t^{\prime} \in[0, t]\right\}
$$

is contained in

$$
\left\{\psi\left(\tau, x_{0}, v\right) \mid \tau \in[0, T] \text { and } \psi\left(\tau^{\prime}, x_{0}, v\right) \in N \text { for all } \tau^{\prime} \in[0, \tau]\right\}
$$


Then it follows that $\mathcal{O}_{\leqslant T^{\prime}}^{N,+}\left(x_{0}\right)$ is contained in

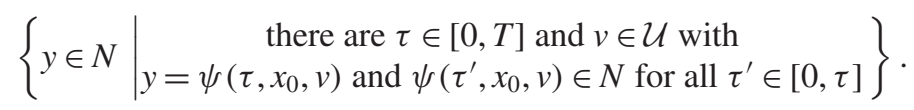

Since by assumption $\mathcal{O}_{\leqslant T^{\prime}}^{N,+}\left(x_{0}\right)$ has non-void interior, also the set in (2.6) has non-void interior. Arguing similarly for negative times, one obtains local accessibility of (2.4).

In order to prove the claim, note first that there is $\delta>0$ such that $\alpha(x) \geqslant \delta>0$ for all $x \in N$, since $\operatorname{cl} N \cap \partial W=\emptyset$. Then it follows for all $v \in \mathcal{U}$ and $\sigma \in \mathbb{R}$

$$
\psi\left(\sigma, x_{0}, v\right) \in N \text { implies } \alpha\left(\psi\left(\sigma, x_{0}, v\right)\right) \geqslant \delta
$$

The proof of Proposition 2.5(ii) shows that for every $u \in \mathcal{U}$ there is $v \in \mathcal{U}$ with

$$
\varphi_{W}\left(t, x_{0}, u\right)=\psi(\tau, x, v), t \quad \in I_{W}\left(x_{0}, u\right),
$$

with $\tau=\rho(t)$ and $v(\tau)=u\left(\rho^{-1}(\tau)\right)$, where $\rho(t):=\int_{0}^{t}\left[\alpha\left(\varphi_{W}\left(s, x_{0}, u\right)\right)\right]^{-1} \mathrm{~d} s, t \in I_{W}\left(x_{0}, u\right)$.

Let $T^{\prime}:=T / \delta$. Then for all $u \in \mathcal{U}$ and all $t \in\left[0, T^{\prime}\right]$, one has that $\varphi\left(t^{\prime}, x_{0}, v\right) \in N$ for all $t^{\prime} \in$ $[0, t]$ implies

$$
\rho(t)=\int_{0}^{t} \frac{1}{\alpha\left(\varphi_{W}\left(s, x_{0}, u\right)\right)} \mathrm{d} s \leqslant T^{\prime} \frac{1}{\delta}=T
$$

and hence $\varphi_{W}\left(t, x_{0}, u\right)=\psi\left(\tau, x_{0}, v\right)$ with $\tau \in[0, T]$ and the claim is proved. Analogous arguments for negative time show that system (2.4) is locally accessible.

Similarly, one argues for the converse implication.

\section{W-control sets}

In this section, the central notion of this paper is defined, subsets of complete approximate controllability which are maximal within the world $W$, and their basic properties are proved. We will rely on the results in Colonius \& Kliemann (2000, Chapter 3) (where global existence of solutions is supposed) and emphasize the new features due to the restriction to $W$.

Definition 3.1 For system (2.1), a set $D \subset W$ with non-void interior is called a $W$-control set if (i) $D \subset \operatorname{cl}_{W} \mathcal{O}^{W,+}(x)$ for all $x \in D$ and (ii) $D$ is maximal with this property, i.e. if $D_{0} \supset D$ has property (i), then $D_{0}=D$.

The $W$-control sets with $W=M$ coincide with the control sets with non-void interior as considered in Colonius \& Kliemann (2000). Non-void interior of a control set (defined as above with $W=M$ ) is not required in Colonius \& Kliemann (2000), but this requirement simplifies many arguments and covers most interesting cases. Hence, we restrict our attention to these sets. In the following, we just speak of control sets when we mean $W$-control sets with $W=M$.

REMARK 3.2 Since approximate controllability holds in a $W$-control set $D$, Colonius \& Kliemann (2000, Proposition 3.2.5) implies that it is contained in a unique control set $D_{M}$. The converse is false: Example 3.5 presents a world without a $W$-control set, whereas there is a control set which has non-void intersection with $W$. 
First we show that the $W$-control sets of system (2.1) coincide with the control sets of system (2.4).

Proposition 3.3 A set $D \subset W$ is a $W$-control set for system (2.1) iff it is a control set for system (2.4).

Proof. This is a consequence of Proposition 2.5(ii): For every $x \in W$, the reachable set from $x$ of system (2.4) coincides with the reachable set $\mathcal{O}^{W,+}(x)$ of system (2.2), hence also their closures with respect to $W$ coincide.

Proposition 3.3 together with Proposition 2.5 shows that all general results in Colonius \& Kliemann (2000, Chapter 3) referring only to controllability properties of control sets are also valid for $W$-control sets. Furthermore, the only difference between the trajectories of (2.1) in $W$ and the trajectories of (2.4) is the parametrization by time: If a trajectory $\varphi\left(t_{k}, x, u\right)$ of (2.4) approaches the boundary of $W$, it follows that $t_{k} \rightarrow \pm \infty$, while the trajectories of (2.1) may approach the boundary of $W$ in finite time. Thus, only the $W$-control sets whose boundary intersects the boundary of $W$ are of independent interest. Recall also that by Proposition 2.10, system (2.4) is locally accessible iff system (2.1) is locally accessible. Then, in particular, for every element $x$ of a $W$-control set $D$ one has int $D \subset \mathcal{O}^{W,+}(x)$. This will be used frequently.

One may be led to think that the $W$-control set can be obtained from a control set by simply intersecting it with the world $W$ and paying some attention to the boundary. But the following example is a $W$-control set with positive distance to the boundary of the world and contained in an invariant control set which is not a subset of $W$.

EXAMPLE 3.4 Let the manifold $M:=\mathbb{R}^{2} \backslash\{0\}$ be parametrized by polar coordinates $(r, \phi)$. The world $W$ is the open right half plane $W:=\{(r, \phi)|| \phi \mid<\pi / 2\}$. Let the system be given by

$$
\begin{aligned}
& \left(\begin{array}{l}
\dot{r} \\
\dot{\phi}
\end{array}\right)=\left(\begin{array}{c}
r(1-r) \\
\sin ^{2}\left(\frac{\phi}{2}\right)
\end{array}\right)+u_{1}(t)\left(\begin{array}{l}
1 \\
0
\end{array}\right)+u_{2}(t)\left(\begin{array}{l}
0 \\
1
\end{array}\right)=:\left(\begin{array}{l}
X_{1}\left(r, \phi, u_{1}\right) \\
X_{2}\left(r, \phi, u_{2}\right)
\end{array}\right), \\
& \left(u_{1}(t), u_{2}(t)\right) \in U:=\left[0, \frac{3}{4}\right) \times\left(-\sin ^{2}\left(\frac{\pi}{8}\right), \sin ^{2}\left(\frac{\pi}{8}\right)\right) .
\end{aligned}
$$

The accessibility rank condition (ARC) holds on $M$. For points $(r, \phi)$ with radius $r \in\left(1, \frac{3}{2}\right)$, one can choose $u_{1}$ such that $X_{1}\left(r, \phi, u_{1}\right)$ is positive, negative or zero. For $r<1, X_{1}\left(r, \phi, u_{1}\right)$ is positive, and for $r>\frac{3}{2}, X_{1}\left(r, \phi, u_{1}\right)$ is negative, independently of the choice of $u_{1}$. If the angle $|\phi|<\pi / 4$, on can choose $u_{2}$ such that $X_{2}\left(r, \phi, u_{2}\right)$ is positive, negative or zero. For all other values of the angle $X_{2}\left(r, \phi, u_{2}\right)>0$, independently of the control. Hence, a $W$-control set $D$ is given by $D:=\{(r, \phi)|| \phi \mid<\pi / 4,1 \leqslant r \leqslant$ $3 / 2\}$.

On the whole state space $M$, the control set $D_{M}$ containing $D$ is given by $D_{M}:=\{(r, \phi) \mid 1 \leqslant r \leqslant$ $\left.\frac{3}{2}, \phi \in[0,2 \pi)\right\}$, which can be seen as follows: Given two points $\left(r_{0}, \phi_{0}\right)$ and $\left(r_{1}, \phi_{0}\right)$ in $C$, one first chooses a control, which keeps $\phi$ constant and steers $\left(r_{0}, \phi_{0}\right)$ to $\left(r_{1}, \phi_{0}\right)$. Then one selects a control that keeps $r$ constant and $X_{2}\left(r, \phi, u_{2}\right)$ constant and positive. By going along the circle with radius $r_{1}$ with constant speed, one reaches $\left(r_{1}, \phi_{1}\right)$. The control set $D_{M}$ is invariant, since it is closed.

Note that $D_{M} \cap W \neq D$ and moreover $d(D, \partial W)>0$. Similar examples can be constructed in systems containing a periodic orbit. 
EXAmple 3.5 Consider the system governed by equation (3.1) with the same world $W$ and controls with values

$$
\left(u_{1}(t), u_{2}(t)\right) \in U:=\left[0, \frac{3}{4}\right) \times\left(0, \sin ^{2}\left(\frac{\pi}{8}\right)\right) .
$$

For every control $u_{2}$, one has $X_{2}\left(r, \phi, u_{2}\right)>0$ and every point in $W$ leaves $W$ eventually flowing counterclockwise. So there is no $W$-control set. Again $D_{M}=\left\{(r, \phi) \mid 1 \leqslant r \leqslant \frac{3}{2}, \phi \in[0,2 \pi)\right\}$ is a control set on $M=\mathbb{R} \backslash\{0\}$.

An important question is the behaviour of $W$-control sets under change of an external parameter $\alpha$. Consider the following family of control systems on $M$ with $u \in \mathcal{U}$ and $\alpha \in A \subset \mathbb{R}^{k}$ :

$$
\dot{x}(t)=X(\alpha, x(t), u(t)), \quad u \in \mathcal{U},
$$

with a smooth map $X: \mathbb{R}^{k} \times M \times \mathbb{R}^{m} \rightarrow T M$ such that $X(\alpha, \cdot, \cdot), \alpha \in \mathbb{R}^{k}$, satisfy the conditions on (2.1). The corresponding solutions are denoted by $\varphi^{\alpha}(t, x, u)$. We suppose that a family of open worlds $W^{\alpha}$ for the $\alpha$-systems is given, while the control range $U$ does not depend on $\alpha$. The following theorem shows that under the Lie algebra rank condition (ARC) the $W$-control sets behave nicely under parameter variation. Recall that a set-valued map $x \mapsto F(x)$ between metric spaces is lower semicontinuous at a point $x_{0}$ if for every open set $U$ with $F\left(x_{0}\right) \cap U \neq \emptyset$ it follows that $F(x) \cap U \neq \emptyset$ for all $x$ in a neighbourhood of $x_{0}$; cf. Aubin \& Frankowska (1990, Definition 1.4.2).

THeOREm 3.6 For the family of systems (3.2), let $\alpha_{0} \in \operatorname{int} A$ and suppose for the open worlds $W^{\alpha} \subset M$ that for every compact set $K \subset W^{\alpha_{0}}$ there is $\varepsilon>0$ with $K \subset W^{\alpha}$ for all $\alpha$ with $\left\|\alpha-\alpha_{0}\right\|<\varepsilon$. Consider a $W^{\alpha_{0}}$-control set $D^{\alpha_{0}}$.

(i) Let $K \subset$ int $D^{\alpha_{0}}$ be a compact set on which the Lie algebra rank condition (ARC) is fulfilled for the parameter value $\alpha_{0}$. Then there is $\delta_{K}>0$ such that for all $\alpha$ with $\left\|\alpha-\alpha_{0}\right\|<\delta_{K}$ there is a unique $W^{\alpha}$-control set $D_{K}^{\alpha}$ with $K \subset$ int $D_{K}^{\alpha}$ for system (3.2) with parameter value $\alpha$.

(ii) Assume that condition (ARC) holds on $W^{\alpha_{0}}$ for the parameter value $\alpha_{0}$. There are $\delta_{0}>0$ and a unique family of $W^{\alpha}$-control sets $D^{\alpha}$ for all $\alpha$ with $\left\|\alpha-\alpha_{0}\right\|<\delta_{0}$ with the following property: For every compact set $K \subset$ int $D^{\alpha_{0}}$, there is a $\delta_{K} \in\left(0, \delta_{0}\right)$ so that $K \subset \operatorname{int} D^{\alpha}$ for every $\alpha$ with $\left\|\alpha-\alpha_{0}\right\|<\delta_{K}$.

(iii) In the situation of (ii), one has that for every open set $U \subset W^{\alpha_{0}}$ with $U \cap \mathrm{cl}_{W} D^{\alpha_{0}} \neq \emptyset$ there is $\delta>0$ such that $U \cap D^{\alpha} \neq \emptyset$ for all $\alpha$ with $\left\|\alpha-\alpha_{0}\right\|<\delta$. In particular, the set-valued maps $\alpha \mapsto D^{\alpha}$ and $\alpha \mapsto \operatorname{cl} D^{\alpha}$ are lower semicontinuous at $\alpha=\alpha_{0}$.

Proof. (i) The proof will show that for all $x, y \in K$ there is $\delta(x, y)>0$ such that $x, y$ are in the interior of a $W^{\alpha}$-control set $D^{\alpha}$ for all $\alpha$ with $\left\|\alpha-\alpha_{0}\right\|<\delta(x, y)$. Then compactness of $K \times K$ will imply the assertion. First we show that $x, y \in K$ are in some control set $W^{\alpha}$ for $\alpha$ close to $\alpha^{0}$.

The accessibility rank condition (cf. Sontag, 1998, Theorem 9 in Chapter 4) implies that there are $u_{x}^{1}, \ldots, u_{x}^{d} \in U$ and $T_{x}>0$ with the following properties: Let $\tau:=\left(\tau_{1}, \ldots, \tau_{d}\right) \in\left(0, T_{x}\right)^{d}$ and define $u \in \mathcal{U}$ by

$$
u(t)=u_{x}^{i} \quad \text { for } t \in\left[-\tau_{1}-\cdots-\tau_{i-1}-\tau_{i},-\tau_{1}-\cdots-\tau_{i-1}\right) .
$$


Then the map $\Psi_{x}:\left(0, T_{x}\right)^{d} \rightarrow M$ given by $\tau \mapsto \varphi^{\alpha_{0}}\left(-\sum \tau_{i}, x, u\right)$ has rank $d$ for all $\tau$. Choose $\tau_{x}$ such that $z_{x}:=\varphi^{\alpha_{0}}\left(-\sum \tau_{i, x}, x, u\right) \in$ int $D^{\alpha_{0}}$. By applying the implicit function theorem to the map

$$
A \times\left(0, T_{x}\right)^{d} \rightarrow M:(\alpha, \tau) \mapsto \varphi^{\alpha}-\sum \tau_{i}, x, u
$$

one finds $\delta_{x}>0$ and a compact neighbourhood $N\left(z_{x}\right)$ of $z_{x}$ with

$$
N\left(z_{x}\right) \subset\left\{\varphi^{\alpha}\left(-\sum \tau_{i}, x, u\right) \mid \tau \in\left(0, T_{x}\right)^{d}\right\} \quad \text { for all } \alpha \text { with }\left\|\alpha-\alpha_{0}\right\| \leqslant \delta_{x} .
$$

We may suppose that $T_{x}>0$ is small enough such that the compact set

$$
\left\{\varphi^{\alpha_{0}}\left(-\sum \tau_{i}, x, u\right) \mid \tau \in\left[0, T_{x}\right]^{d}\right\} \subset W^{\alpha_{0}} .
$$

Hence, there is $\delta>0$ such that it is contained in every set $W^{\alpha}$ with $\left\|\alpha-\alpha_{0}\right\|<\delta$. By continuity of $\varphi^{\alpha}$ with respect to $\alpha$, it follows that we may take $\delta_{x}>0$ small enough such that for every $\alpha$ with $\left\|\alpha-\alpha_{0}\right\| \leqslant \delta_{x}$

$$
N\left(z_{x}\right) \subset\left\{\varphi^{\alpha}\left(-\sum \tau_{i}, x, u\right) \mid \tau \in\left[0, T_{x}\right]^{d}\right\} \subset W^{\alpha} .
$$

The same construction for $y$ shows that there are $u_{y}^{1}, \ldots, u_{y}^{d} \in U$, numbers $T_{y}, \tau_{y}>0$ and a neighbourhood $N\left(z_{y}\right)$ of $z_{y}:=\varphi^{\alpha_{0}}\left(\sum \tau_{i, y}, x, u\right) \in \operatorname{int} D^{\alpha_{0}}$ as well as $\delta_{y}>0$ with the following property: for every $\alpha$ with $\left\|\alpha-\alpha_{0}\right\| \leqslant \delta_{y}$

$$
N\left(z_{y}\right) \subset\left\{\varphi^{\alpha}\left(-\sum \tau_{i, y}, y, u\right) \mid \tau \in\left[0, T_{y}\right]^{d}\right\} \subset W^{\alpha} .
$$

We may suppose that $\delta_{1}(x, y):=\delta_{y}=\delta_{x}$ and take $T:=T_{x}=T_{y}>0$ small enough such that $N\left(z_{x}\right), N\left(z_{y}\right) \subset$ int $D^{\alpha_{0}}$ since $x, y \in \operatorname{int} D^{\alpha_{0}}$. In particular, $z_{x}, z_{y} \in \operatorname{int} D^{\alpha_{0}}$ and there are controls $u_{1}, u_{2} \in \mathcal{U}$ and times $S_{1}, S_{2}>0$ with

$$
\varphi_{W^{\alpha_{0}}}^{\alpha_{0}}\left(S_{1}, x, u_{1}\right)=z_{y} \quad \text { and } \quad \varphi_{W^{\alpha_{0}}}^{\alpha_{0}}\left(S_{2}, y, u_{2}\right)=z_{x} .
$$

By continuous dependence on the parameter $\alpha$, there exists $\delta_{2}(x, y)$ with $0<\delta_{2}(x, y) \leqslant \delta_{1}(x, y)$ such that for all $\alpha$ with $\left\|\alpha-\alpha_{0}\right\|<\delta_{2}$

$$
\varphi^{\alpha}\left(S_{1}, x, u_{1}\right) \in N\left(z_{y}\right) \quad \text { and } \quad \varphi^{\alpha}\left(S_{2}, y, u_{2}\right) \in N\left(z_{x}\right) .
$$

As above, we may assume that the trajectories $\varphi^{\alpha}\left(t, x, u_{1}\right), t \in\left[0, S_{1}\right]$ and $\varphi^{\alpha}\left(t, y, u_{2}\right), t \in\left[0, S_{2}\right]$, are in $W^{\alpha}$. Concatenating the corresponding controls, one finds that this construction already shows that $x$ and $y$ are in $W^{\alpha}$-control sets $D^{\alpha}$ for all $\alpha$ with $\left\|\alpha-\alpha_{0}\right\| \leqslant \delta_{2}(x, y)$. We have to refine the construction slightly, in order to show that $x$ and $y$ are in the interior of $D^{\alpha}$.

Use again the accessibility rank condition, now for positive time, in order to find $u^{1}, \ldots, u^{d} \in U$ and $T>0$ with the following properties: Let $\tau:=\left(\tau_{1}, \ldots, \tau_{d}\right) \in(0, T)^{d}$ and define $u^{+} \in \mathcal{U}$ by

$$
u^{+}(t)=u^{i} \quad \text { for } t \in\left[\tau_{1}+\cdots+\tau_{i-1}, \tau_{1}+\cdots+\tau_{i-1}+\tau_{i}\right) .
$$

Then the map $\Psi:(0, T)^{d} \rightarrow M$ given by $\tau \mapsto \varphi^{\alpha_{0}}\left(\sum \tau_{i}, y, u\right)$ has rank $d$ for all $\tau$. Choose $\tau^{+}$such that $z^{+}:=\varphi^{\alpha_{0}}\left(\sum \tau_{i}^{+}, y, u^{+}\right) \in \operatorname{int} D^{\alpha_{0}}$. By applying the implicit function theorem to the map

$$
A \times(0, T)^{d} \rightarrow M:(\alpha, \tau) \mapsto \varphi^{\alpha}\left(\sum \tau_{i}, y, u^{+}\right)
$$


one finds $\delta^{+}>0$ and a neighbourhood $N\left(z^{+}\right)$of $z^{+}$with $N\left(z^{+}\right) \subset$ int $D^{\alpha_{0}}$ and

$$
N\left(z^{+}\right) \subset\left\{\varphi^{\alpha}\left(\sum \tau_{i}, y, u^{+}\right) \mid \tau \in(0, T)^{d}\right\} \quad \text { for all } \alpha \text { with }\left\|\alpha-\alpha_{0}\right\|<\delta^{+} .
$$

Since there are $S_{3}>0$ and $u_{3} \in \mathcal{U}$ with $\varphi^{\alpha_{0}}\left(S_{3}, z^{+}, u_{3}\right)=z_{x}$, we may assume that $N\left(z^{+}\right) \subset \mathcal{O}^{\alpha,-}(x)$ for $\left\|\alpha-\alpha_{0}\right\|<\delta^{+}$. Concatenating the corresponding controls, one concludes that all points in $N\left(z^{+}\right)$can be steered to $x$ and then to $y$, and $x$ and $y$ can be steered to every point in $N\left(z^{+}\right)$by trajectories of the $\alpha$-system. The corresponding trajectories remain in $W^{\alpha}$, hence there is a $W^{\alpha}$-control set $D^{\alpha}$ with $x, y \in D^{\alpha}$ and $N\left(z^{+}\right) \subset D^{\alpha}$. Since $x$ can be reached from a point in the interior of $D^{\alpha}$, it follows that $x \in \operatorname{int} D^{\alpha}$ (this is a standard property of control sets which follows by continuous dependence on initial values). This holds for all $\alpha$ with $\left\|\alpha-\alpha_{0}\right\|<\delta(x, y)$. Since $K$ is compact, one finds finitely many points $x_{1}, \ldots, x_{n} \in K$ and $\delta_{1}, \ldots, \delta_{n}>0$ such that every $x \in K$ is in some control set $D^{\alpha}(x)$ with $\left\|\alpha-\alpha_{0}\right\|<\delta_{i}$. Thus, for all $\alpha$ with $\left\|\alpha-\alpha_{0}\right\|<\min _{i=1, \ldots, n} \delta_{i}$ every point $x \in K$ is in one of the control sets $D^{\alpha}\left(x_{i}\right)$ and the construction above shows that all $D^{\alpha}\left(x_{i}\right)$ coincide, if $\left\|\alpha-\alpha_{0}\right\|$ is small enough.

(ii) Since $W^{\alpha_{0}}$ has a countable base, one finds a sequence of compact sets $K_{n}$ with $K_{n} \subset$ int $K_{n+1}$ and $\bigcup_{n \in \mathbb{N}} K_{n}=\operatorname{int} D^{\alpha_{0}}$, cf. Berger \& Gostiaux (1988, Lemma 3.2.6). If $K$ is a compact set with $K \subset \operatorname{int} D^{\alpha_{0}}$, there is $n$ such that $K \subset K_{n}$. In fact, since for every $x \in K$ there is $m \in \mathbb{N}$ with $x \in K_{m} \subset$ int $K_{m+1}$, we find a neighbourhood $N(x) \subset$ int $K_{m+1} \subset$ int $D^{\alpha_{0}}$. By compactness of $K$, finitely many $N(x)$ already cover $K$ and hence $K \subset K_{n}$ for some $n \in \mathbb{N}$.

By assertion (i), one finds $\delta_{n}>0$ such that there are $W^{\alpha}$-control sets $D_{n}^{\alpha}$ with

$$
K_{n} \subset \operatorname{int} D_{n}^{\alpha} \quad \text { for all } \alpha \text { with }\left\|\alpha-\alpha_{0}\right\|<\delta_{n} .
$$

Since $K \subset K_{n}$ for all $n$, it follows that the $W^{\alpha}$-control sets $D_{n}^{\alpha}$ and $D_{m}^{\alpha}$ coincide for $\alpha<\min \left(\delta_{n}, \delta_{m}\right)$, and hence we may write them as $D^{\alpha}$. The family of control sets $D^{\alpha}$ with $\left\|\alpha-\alpha_{0}\right\|<\delta:=\delta_{1}$ has the desired property.

For $\left\|\alpha-\alpha_{0}\right\|$ small enough, the family $D^{\alpha}$ is unique. In fact, otherwise, there are a sequence $\alpha_{m} \rightarrow$ $\alpha_{0}$ and control sets $D^{\alpha_{m}} \neq \tilde{D}^{\alpha_{m}}$ and a compact set $K \subset \operatorname{int} D^{\alpha_{0}}$ such that $K \subset \operatorname{int} D^{\alpha_{m}}$ and $K \subset \operatorname{int} \tilde{D}^{\alpha_{m}}$ for $m$ large enough. This cannot be.

(iii) Let $U \subset W^{\alpha_{0}}$ be an open set with $U \cap \mathrm{cl}_{W} D^{\alpha_{0}} \neq \varnothing$. By (ARC), the closure of a control set coincides with the closure of its interior. Hence, Proposition 3.3 implies $\mathrm{cl}_{W} D^{\alpha_{0}}=\mathrm{cl}_{W}$ int $D^{\alpha_{0}}$, and it follows that $U \cap \operatorname{int} D^{\alpha_{0}} \neq \varnothing$ and one finds a compact set $K \subset U \cap$ int $D^{\alpha_{0}}$. Hence, by (ii) there is $\varepsilon>0$ such that for all $\alpha$ with $\left\|\alpha-\alpha_{0}\right\|<\varepsilon$, there is a $W^{\alpha}$-control set $D^{\alpha}$ with $K \subset \operatorname{int} D^{\alpha}$. It follows that $U \cap D^{\alpha} \neq \emptyset$ and also the lower semicontinuity properties are shown.

We note the following corollary.

COROLlary 3.7 If system (3.2) is locally accessible on $W$ for all $\alpha$ in a neighbourhood of $\alpha_{0}$, then for all $\alpha$ with $\left\|\alpha-\alpha_{0}\right\|$ small enough there are $W$-control sets $D^{\alpha}$ with int $D^{\alpha_{0}} \cap \operatorname{int} D^{\alpha} \neq \emptyset$.

Proof. By Colonius \& Kliemann (2000, Theorem A.4.5), local accessibility implies that the Lie algebra rank condition (ARC) holds on an open and dense subset of $W$. (This theorem applies here, since local accessibility in the sense of Definition 2.6 implies the weaker version of local accessibility used in Colonius \& Kliemann (2000), cf. Remark 2.7.) Hence, there is a point $x \in \operatorname{int} D^{\alpha_{0}}$ where (ARC) holds. Theorem 3.6(i) shows that there is $\delta>0$ such that for $\left\|\alpha-\alpha_{0}\right\|<\delta$ there is a control set $D^{\alpha}$ with $x \in \operatorname{int} D^{\alpha}$. 
REMARK 3.8 A different approach to parameter dependence is found in Gayer (2004) and Graf (2011), where the parameter influences the control range $U$.

\section{Relatively invariant $W$-control sets}

This section discusses $W$-control sets which are invariant with respect to $W$ and hence deserve special interest.

First recall from Colonius \& Kliemann (2000) that an invariant control set for a system of the form (2.1) is a control set $C$ with $\operatorname{cl} C=\operatorname{clO}^{+}(x)$ for all $x \in C$. If the system is locally accessible and the solutions are defined on $\mathbb{R}$, the invariant control sets are the closed control sets, cf. Colonius $\&$ Kliemann (2000, Theorem 3.1.5). Next we define a generalization for $W$-control sets.

Definition 4.1 A $W$-control set $C$ for system (2.1) is called relatively invariant, if

$$
\mathrm{cl}_{W} C=\operatorname{cl}_{W} \mathcal{O}^{W,+}(x) \text { for all } x \in C \text {. }
$$

It is an immediate consequence of the definitions and Proposition 3.3 that the relatively invariant $W$-control sets for system (2.1) coincide with the invariant control sets of system (2.4). Hence, the relatively invariant $W$-control sets are the control sets which are closed relative to $W$, i.e. $\mathrm{cl}_{W} C=C$, if system (2.4) is locally accessible.

Next we show that a relatively invariant $W$-control set that is bounded away from the boundary of the world is itself a invariant control set on the whole manifold $M$.

Proposition 4.2 Let $C$ be a relatively invariant $W$-control set and assume that system (2.1) is locally accessible on $W$. Then $C$ is an invariant control set in the state space $M$ iff $\partial W=\emptyset$ or there is $\delta>0$ with $\operatorname{dist}(x, \partial W) \geqslant \delta$ for every $x$ in $C$.

Proof. If $C$ is an invariant control set for the system in $M$, the subset $C$ of the open set $W$ is closed, hence compact in $\mathrm{cl} W$ and in $M$. Thus, there is $\delta>0$ with $\operatorname{dist}(x, \partial W) \geqslant \delta$ for every $x \in C$. Conversely, suppose that $\delta>0$ with $\operatorname{dist}(x, \partial W) \geqslant \delta$ for every $x \in C$. Since $C=\operatorname{cl}_{W} \mathcal{O}^{W,+}(x)$ for every $x \in C$, it follows that $\operatorname{dist}(y, \partial W) \geqslant \delta$ for every $y \in \operatorname{cl}_{W} \mathcal{O}^{W,+}(x)$. Thus, $\operatorname{clO}^{+}(x)=\operatorname{cl}_{W} \mathcal{O}^{W,+}(x)=C$ for every $x \in C$ showing that $C$ is an invariant control set in the state space $M$.

The following proposition gives a criterion for checking relative invariance.

Proposition 4.3 Assume that system (2.1) is locally accessible from all points in the closure of a $W$-control set $D$. Then $D$ is relatively invariant iff $D$ is closed relative to $W$, i.e. $\operatorname{cl}_{W} D=D$.

Proof. Let $D$ be relatively invariant and consider $x \in \operatorname{cl}_{W} D$. Then $\mathcal{O}^{W,+}(x) \subset \operatorname{cl}_{W} C$, since otherwise continuous dependence on initial values implies that there are $y \in C$ and $t>0$ with $\varphi(t, y, u) \in$ $\mathcal{O}^{W,+}(y) \backslash \operatorname{cl}_{W} C$ contradicting relative invariance. By local accessibility int $\mathcal{O}^{W,+}(x) \neq \emptyset$, hence there is a point $z \in \operatorname{int} \mathcal{O}^{W,+}(x) \cap C$. Using the definition of relatively invariant $W$-control sets, we find $\mathrm{cl}_{W} C=\operatorname{cl}_{W} \mathcal{O}^{+}(z) \subset \operatorname{cl}_{W} \mathcal{O}^{+}(x)$ and $\operatorname{cl}_{W} C=\operatorname{cl}_{W} \mathcal{O}^{+}(x)$ follows. Now the maximality property of $W$ control sets implies $x \in C$ showing that $D$ is closed in $W$. The converse follows as in Colonius \& Kliemann (2000, Theorem 3.2.20). 
Next we will analyse the existence and the number of relatively invariant $W$-control sets. The next theorem follows as Colonius \& Kliemann (2000, Proposition 3.3.7 and Theorem 3.3.10) where a similar situation is considered and hence the proof will be omitted.

THEOREM 4.4 Suppose that system (2.1) is locally accessible in the world $W$.

(i) Fix $x \in W$ and assume that there exists a set $Q \subset W$ which is closed in $M$ such that $\mathrm{cl}_{W} \mathcal{O}^{W,+}(y) \cap Q \neq \emptyset$ for all $y \in \mathcal{O}^{W,+}(x)$. Then there exists a relatively invariant $W$-control set $C \subset \operatorname{cl}_{W} \mathcal{O}^{W,+}(x)$.

(ii) The following assertions are equivalent:

(a) There is a set $Q \subset W$ which is closed in $M$ such that $Q \cap \operatorname{cl}_{W} \mathcal{O}^{W,+}(x) \neq \emptyset$ for every $x \in W$.

(b) For every $x \in W$, there is a relatively invariant $W$-control set $C$ contained in $\operatorname{cl}_{W} \mathcal{O}^{W,+}(x)$ and there are only finitely many relatively invariant $W$-control sets.

The following example shows that the number of relatively invariant $W$-control sets in a world $W$ may be infinite.

EXAMPLE 4.5 Let $W:=(0,1) \subset M:=\mathbb{R}$ and consider

$$
\dot{x}(t)=x \sin \frac{1}{x}+x u=: X(x, u) \quad \text { with } u \in U:=\left(-\sin \left(\frac{\pi}{4}\right), \sin \left(\frac{\pi}{4}\right)\right) .
$$

Note that accessibility rank condition (ARC) holds on $W$. The sets

$$
C_{k}:=\left[\left(2 k \pi+\frac{\pi}{4}\right)^{-1},\left(2 k \pi-\frac{\pi}{4}\right)^{-1}\right], \quad k \in \mathbb{N}
$$

are relatively invariant $W$-control sets. These $W$-control sets are in fact invariant control sets in $M=\mathbb{R}$. They cluster at the boundary of $W$ (they are separated by control sets which are not invariant).

In order to analyse the behaviour of infinitely many relatively invariant $W$-control sets, we adapt the following lemma from Colonius \& Kliemann (2000, Lemma 4.5.4).

Lemma 4.6 Let $x \in W$ and $u \in \mathcal{U}$ with $\varphi(T, x, u) \in \operatorname{int} \mathcal{O}_{\leqslant T+S}^{W,+}(x)$ for some $T, S>0$ and assume that the system is locally accessible at $\varphi(T, x, u)$. Then

$$
x \in \operatorname{int} \mathcal{O}_{\leqslant T+2 S}^{W,-}(\varphi(T, x, u)) .
$$

Proof. We find an open neighbourhood $N(y) \subset \operatorname{int} \mathcal{O}_{\leqslant T+S}^{W,+}(x)$ of $y:=\varphi(T, x, u)$. Local accessibility at $y$ implies that there is $z \in N(y) \cap \operatorname{int} \mathcal{O}_{\leqslant t_{0}}^{W,-}(y)$ for every $t_{0}$ with $0<t_{0} \leqslant S$. Then there are a control $u$ and a neighbourhood $N(x)$ of $x$ such that $N(x)$ is mapped in a time $T_{1} \leqslant T+S$ via the solution map corresponding to $u$ onto a neighbourhood $N(z)$ of $z$ contained in $N(y) \cap \mathcal{O}_{\leqslant}^{W,-}(y)$. We obtain

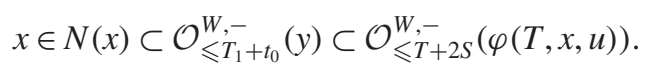

The following theorem shows that infinitely many relatively invariant $W$-control sets can only cluster at the boundary of the world $W$. 
TheOREM 4.7 Suppose that $C_{n}, n \in \mathbb{N}$, are countably many pairwise distinct relatively invariant $W$-control sets for system (2.1) on an open, relatively compact world $W$ with $\partial W \neq \emptyset$. If the system is locally accessible on $W$, then $d_{H}\left(C_{n}, \partial W\right) \rightarrow 0$ in the Hausdorff metric for $n \rightarrow \infty$.

Proof. If the assertion is false, one finds $\delta>0$ and $n_{k} \rightarrow \infty$ such that the $W$-control sets $C_{n_{k}}$ are pairwise different and there are $x_{k} \in C_{n_{k}}$ with $\operatorname{dist}\left(x_{k}, \partial W\right) \geqslant \delta$. This sequence has a cluster point and every cluster point $x$ satisfies $\operatorname{dist}(x, \partial W) \geqslant \delta$, so $x \in W$. By local accessibility in $W$, there are $T, S>0$ and a control $u$ with $\varphi(T, x, u) \in \operatorname{int} \mathcal{O}_{\leqslant T+S}^{W,+}(x) \subset W$. By Lemma 4.6, we find that $x \in \operatorname{int} \mathcal{O}_{\leqslant T+2 S}^{W,-}(\varphi(T, x, u))$. Hence, for $k \in \mathbb{N}$ large enough one has

$$
x_{k} \in \mathcal{O}_{\leqslant T+2 S}^{W,-}(\varphi(T, x, u)) .
$$

This shows that for all $k$ large enough one finds that the point $x_{k} \in C_{n_{k}}$ can be steered to the single point $\varphi(T, x, u) \in W$. This contradicts relative invariance of the pairwise different $W$-control sets $C_{n_{k}}$.

The following examples further illustrate the difference between $W$-control sets and control sets in the whole state space. The first example presents a $W$-invariant control set which is variant on the whole manifold and the second presents a $W$-control set which is not relatively invariant and contained in an invariant control set.

EXAmple 4.8 Consider on $M:=\mathbb{R}$ with world $W:=(-1,1)$, the system

$$
\dot{x}=x+u \quad \text { with } u(t) \in U:=[-1,1] .
$$

As one can easily see, the system is controllable on $W$, hence $W$ itself is a relatively invariant $W$-control set. The set $W$ is also a control set in the whole state space $\mathbb{R}$. In fact, if $x \geqslant 1$, then $x+u \geqslant 0$ for all $u$ and the reachable set is $\mathcal{O}^{+}(x)=[x, \infty)$. Analogously, one finds that for $x \leqslant-1$ the reachable set is $\mathcal{O}^{+}(x)=(-\infty,-x]$. The set $W$ is not an invariant control set, since for $x \in(-1,1)$ the reachable set is $\mathcal{O}^{+}(x)=\mathbb{R}$.

EXAmple 4.9 The set $D_{M}$ in Example 3.4 is an invariant control set on the whole manifold, whereas $D$ is a $W$-control set which is not relatively invariant.

EXAMPLE 4.10 Let $D$ be an arbitrary control set on $M$ for a system of the form (2.1) and define the world $W$ as the domain of attraction of $D$, i.e.

$$
W:=\left\{y \in M \mid \mathcal{O}^{+}(y) \cap \operatorname{int} D \neq \emptyset\right\} .
$$

Then $D$ is a relatively invariant $W$-control set. In fact, from every $x \in D$ one can reach approximately every $y \in D$ by a trajectory in $D \subset W$, thus $D \subset \operatorname{cl}_{W} \mathcal{O}^{W,+}(x)$ showing that $D$ is a $W$-control set. Furthermore, if $y \in \mathcal{O}^{W,+}(x) \subset \mathcal{O}^{+}(x) \cap W$, then $\mathcal{O}^{+}(y) \cap$ int $D \neq \emptyset$, and hence maximality of $D$ as a control set implies that $y \in D$. Hence, $\mathcal{O}^{W,+}(x) \subset D$ for every $x \in D$ and relative invariance follows.

Finally, we discuss the behaviour of invariant control sets which lose their invariance under parameter variation. For this, we change the perspective: Now the worlds $W^{\alpha}$ are no longer taken as given, but are constructed. Recall that a neighbourhood $N$ of a compact set $K$ is a set containing $K$ in its interior with $d_{H}(N, K)>0$.

Lemma 4.11 Consider a family of systems of the form (3.2) defined on $M$ and let $\alpha_{0} \in \operatorname{int} A$. For $\alpha=\alpha_{0}$, suppose that $D^{\alpha_{0}}$ is a compact invariant control set in $M$ and accessibility rank condition (ARC) holds on $D^{\alpha_{0}}$. Fix a point $x_{0} \in \operatorname{int} D^{\alpha_{0}}$. Then there are a neighbourhood $N$ of $D^{\alpha_{0}}$ and a constant $\delta_{0}>0$ such that 
for every $\alpha$ with $\left\|\alpha-\alpha_{0}\right\|<\delta_{0}$ there are a world $W^{\alpha}$ with $N \subset W^{\alpha}=\mathcal{O}^{W^{\alpha},-}\left(x_{0} ; \alpha\right)$ and a $W^{\alpha}$-control set $D^{\alpha}$ for the $\alpha$-system depending lower semicontinuously on $\alpha$ in $\alpha_{0}$. For all compact subsets $K \subset \operatorname{int} D^{\alpha_{0}}$, there is $\delta_{K} \in\left(0, \delta_{0}\right)$ with $K \subset$ int $D^{\alpha}$ for $\left\|\alpha-\alpha_{0}\right\|<\delta_{K}$.

Proof. First observe that the accessibility rank condition and hence local accessibility holds on a neighbourhood of $D^{\alpha_{0}}$ for all $\alpha$ near $\alpha_{0}$ by the continuity assumption. By local accessibility and compactness of $D^{\alpha_{0}}$, there is $T>0$ such that for every $y \in D^{\alpha_{0}}$ there are $t(y) \in(0, T]$ and a control $u(y) \in \mathcal{U}$ with $\varphi^{\alpha_{0}}(t, y, u(y)) \in D^{\alpha_{0}}$ for $t \in(0, T]$ and $\varphi^{\alpha_{0}}(t(y), y, u(y))=x_{0}$. Take a compact subset $K \subset \operatorname{int} D^{\alpha_{0}}$ containing $x_{0}$ in its interior. By continuous dependence on the initial value, one finds for every $y \in D^{\alpha_{0}}$ a number $\varepsilon(y)>0$ and an open neighbourhood $N(y)$ such that for $\left\|\alpha-\alpha_{0}\right\|<\varepsilon(y)$ one has $\varphi^{\alpha}(\tau(y), N(y), u(y)) \subset K$. Finitely many of these neighbourhoods $N\left(y_{i}\right), i=1, \ldots, n$, cover $D^{\alpha_{0}}$. Then $N:=\bigcup_{i=1}^{n} N\left(y_{i}\right)$ is an open neighbourhood of $D^{\alpha_{0}}$ and we define $W^{\alpha_{0}}:=N$. Then, naturally, $D^{\alpha_{0}} \subset W^{\alpha_{0}}$ is a $W^{\alpha_{0}}$-control set.

For $\alpha \neq \alpha_{0}$ with $\left\|\alpha-\alpha_{0}\right\|<\varepsilon_{0}:=\min _{i=1, \ldots, n} \varepsilon\left(y_{i}\right)$, let a world $W^{\alpha}$ for the $\alpha$-system be defined by

$$
W^{\alpha}:=\bigcup_{i=1}^{n}\left\{\varphi^{\alpha}\left(t, y, u_{y_{i}}\right) \mid y \in N\left(y_{i}\right) \text { and } t \in\left[0, \tau\left(y_{i}\right)\right]\right\} .
$$

In fact, all sets $W^{\alpha}$ are open and connected with compact closure and contain $N=W^{\alpha_{0}}$. Thus, all assumptions of Theorem 3.6 are fulfilled. It follows that for $\varepsilon_{0}>0$ small enough there is a family of $W^{\alpha}$ control sets $D^{\alpha}$ with $K \subset \operatorname{int} D^{\alpha}$. Then controllability in the interior of $D^{\alpha}$ implies that $x_{0} \in \mathcal{O}^{W^{\alpha},+}(y ; \alpha)$ for all $y$ in $W^{\alpha}$, i.e. $W^{\alpha} \subset \mathcal{O}^{W^{\alpha},-}\left(x_{0} ; \alpha\right)$.

Furthermore, Theorem 3.6(iii) shows lower semicontinuity of $D^{\alpha}$ in $\alpha=\alpha_{0}$.

The following theorem is a main result of this paper. It gives conditions which ensure that an invariant control set $C^{\alpha_{0}}$ in $M$ turns into $W$-control sets $C^{\alpha}$ which are relatively invariant. Thus, this result gives insight into the behaviour of control sets when their invariance is lost.

Theorem 4.12 Consider a family of systems of the form (3.2) and suppose that $C^{\alpha_{0}}$ is a compact invariant control set in the state space $M$ and that for $\alpha_{0}$ accessibility rank condition (ARC) holds on $C^{\alpha_{0}}$. Then there are $\delta_{0}>0$ and a neighbourhood $N$ of $C^{\alpha_{0}}$ such that for $\alpha$ with $\left\|\alpha-\alpha_{0}\right\|<\delta_{0}$ the $\alpha$-systems have the following property: there are a family of worlds $W^{\alpha}$ containing $N$ and relatively invariant $W^{\alpha}$-control sets $C^{\alpha}$ depending lower semicontinuously on $\alpha$ in $\alpha_{0}$ such that for all compact subsets $K \subset$ int $C^{\alpha_{0}}$ there is $\delta_{K} \in\left(0, \delta_{0}\right)$ with $K \subset \operatorname{int} C^{\alpha}$ for $\left\|\alpha-\alpha_{0}\right\|<\delta_{K}$.

Proof. It only remains to show that the $W^{\alpha}$-control sets $C^{\alpha}:=D^{\alpha}$ constructed in the proof of Lemma 4.11 are relatively invariant for $\alpha$ close to $\alpha_{0}$. If the $W^{\alpha}$-control set $D^{\alpha}$ is not relatively invariant, there are $x_{1} \in D^{\alpha}$ and $u^{\alpha} \in \mathcal{U}$ with $\varphi_{W^{\alpha}}^{\alpha}\left(t^{\alpha}, x_{1}, u^{\alpha}\right) \in W^{\alpha} \backslash \operatorname{cl}_{W^{\alpha}} D^{\alpha}$. Choose a point $x_{0} \in \operatorname{int} D^{\alpha_{0}}$. In the proof of Lemma 4.11, it was shown that $x_{0} \in D^{\alpha}$. Hence, approximate controllability in the interior of a control set and continuous dependence on the initial value imply that we may take $x_{1}=x_{0}$. Since $W^{\alpha} \subset \mathcal{O}^{W^{\alpha},-}\left(x_{0} ; \alpha\right)$, this implies the contradiction $\varphi_{W^{\alpha}}^{\alpha}\left(t^{\alpha}, x_{0}, u^{\alpha}\right) \in D^{\alpha}$.

Compare the assertion of Theorem 4.12 to Theorem 3.6 where another lower semicontinuity result or arbitrary $W^{\alpha}$-control sets was given.

REMARK 4.13 If the control sets $D^{\alpha}$ are invariant control sets in $M$, they may depend continuously on $\alpha$. If the relatively invariant $W^{\alpha}$-control sets $D^{\alpha}$ are not invariant control sets, they have non-void intersection with the boundary of $W^{\alpha}$, and hence the map $\alpha \mapsto \operatorname{cl} C^{\alpha}$ is discontinuous in $\alpha=\alpha_{0}$ with 
respect to the Hausdorff metric, since $N \subset W^{\alpha}$ and $N$ is a neighbourhood of $C^{\alpha_{0}}$. This illustrates the interest of this result for the understanding of the loss of invariance.

REMARK 4.14 For control ranges depending monotonously on a scalar parameter $\alpha$, Gayer (2004, Corollary 24) has shown that invariant control sets remain invariant if they change continuously (in the Hausdorff metric). On the other hand, Theorem 4.12 implies that conversely invariant control sets which become variant change discontinuously and become relatively invariant $W^{\alpha}$-control sets for appropriately defined worlds $W^{\alpha}$.

The worlds $W^{\alpha}$ constructed above and hence the associated relatively invariant $W^{\alpha}$-control sets $C^{\alpha}$ certainly are not unique (the $C^{\alpha}$ are unique for given $W^{\alpha}$ ). Furthermore, the worlds $W^{\alpha}$ are constructed locally around $C^{\alpha_{0}}$. Hence, one may ask, if one may construct worlds and corresponding relatively invariant control sets which are unique. It is easy to see that for worlds $W_{1}, W_{2}$ with relatively invariant $W_{i}$-control sets $C_{i}$ satisfying $C_{1} \cap C_{2} \neq \emptyset$ the union $C_{1} \cup C_{2}$ is a $\left(W_{1} \cup W_{2}\right)$-control set which, however, may not be relatively invariant (e.g. one may go from $C_{1}$ into $W_{2}$ ). Nevertheless, if the worlds increase monotonically, the following proposition shows that maximal relatively invariant $W$-control sets exist.

Proposition 4.15 Let $W_{i}, i \in I$, where $I$ is an index set, be a family of worlds contained in a compact set $K \subset M$, and suppose that the worlds are linearly ordered with respect to set inclusion, i.e. $W_{i} \subset W_{j}$ or $W_{j} \subset W_{i}$ for all $i, j \in I$. Let $C_{i}$ be relatively invariant $W_{i}$-control sets with $\bigcap_{i \in I} \operatorname{int} C_{i} \neq \emptyset$. Then $C:=$ $\bigcup_{i \in I} C_{i}$ is a relatively invariant $W$-control set for the world $W:=\bigcup_{i \in I} W_{i}$.

Proof. Since $W_{i} \subset K$ for all $i$, it follows that $W$ is relatively compact. Furthermore, $W$ is open and connected, thus $W$ is a world. It is easily seen that approximate in $C$ controllability holds and that $C$ is a maximal set with this property. It remains to show that $C$ is relatively invariant: So suppose that $x_{0} \in C$ and $\varphi\left(t_{0}, x_{0}, u_{0}\right) \in W \backslash C$ where $t_{0}>0, u_{0} \in \mathcal{U}$ and $\varphi\left(t, x_{0}, u_{0}\right) \in C$ for $t \in\left[0, t_{0}\right)$. Then there are indices $i, j \in I$ such that $x_{0} \in C_{i}$ and $\varphi\left(t_{0}, x_{0}, u_{0}\right) \in W_{j} \backslash C_{j}$. Then either $W_{i} \subset W_{j}$ or $W_{j} \subset W_{i}$. In the first case, $x_{0} \in C_{j}$. Since by Proposition 4.3 , the set $C_{j}$ is closed in $W$ there is $t_{1} \in\left(0, t_{0}\right)$ with $\varphi\left(t_{1}, x_{0}, u_{0}\right) \in$ $W_{j} \backslash \mathrm{cl} C_{j}$ contradicting relative invariance of $C_{j}$ in $W_{j}$. In the second case, $\varphi\left(t_{0}, x_{0}, u_{0}\right) \in W_{i} \backslash C_{i}$ and the same arguments again yield a contradiction.

The following one-dimensional example illustrates the behaviour of control sets and $W$-control sets as described in Theorem 4.12.

EXAMPLE 4.16 Consider the parameter-dependent system on $\mathbb{R}$ given by

$$
\dot{x}=x^{2}-x+\alpha u
$$

with $u(t) \in U:=[0,1]$ and $\alpha \in(0, \infty)$.

For $u=0$, one has $x^{2}-x+\alpha u=x^{2}-x<0$ iff $x \in(0,1)$. For $u \in[0,1]$ and $\frac{1}{4}-\alpha u \geqslant 0$, one has $x^{2}-x+\alpha u=\left(x-\frac{1}{2}\right)^{2}-\frac{1}{4}+\alpha u>0$ iff $x>\frac{1}{2} \pm \sqrt{\frac{1}{4}-\alpha u}$ and $\frac{1}{4}-\alpha u \geqslant 0$. Now $\frac{1}{4}-\alpha u \geqslant 0$ iff $u \leqslant \frac{1}{4 \alpha}$.

Let $\alpha \leqslant \frac{1}{4}$. Then two control sets in $M=\mathbb{R}$ exist:

$$
C_{M}^{\alpha}=\left[0, \frac{1}{2}-\sqrt{\frac{1}{4}-\alpha}\right] \quad \text { and } \quad D_{M}^{\alpha}=\left(\frac{1}{2}+\sqrt{\frac{1}{4}-\alpha}, 1\right)
$$


Note that $C_{M}^{\alpha}$ is an invariant control set, while $D_{M}^{\alpha}$ is not invariant. For $\alpha=\frac{1}{4}$, the closure of $D_{M}^{1 / 4}=$ $\left(\frac{1}{2}, 1\right)$ intersects $C_{M}^{1 / 4}=\left[0, \frac{1}{2}\right]$. For $\alpha>\frac{1}{4}$, the two control sets merge and give a single control set $D_{M}^{\alpha}=$ $[0,1)$ which is not invariant.

Theorem 4.12 can be applied with $\alpha_{0}=\frac{1}{4}$ to the invariant control set $C^{1 / 4}:=C_{M}^{1 / 4}=\left[0, \frac{1}{2}\right]$. Let $N:=(a, b)$ be a neighbourhood of $C^{1 / 4}$ with $a<0$ and $\frac{1}{2}<b<1$. For $\alpha>\frac{1}{4}$, consider a world $W^{\alpha}:=\left(c^{\alpha}, d^{\alpha}\right)$ with $c^{\alpha} \leqslant a$ and $b \leqslant d^{\alpha}<1$. Then a relatively invariant $W^{\alpha}$-control set is given by $D^{\alpha}:=\left[0, d^{\alpha}\right)$. Clearly, $D^{\alpha} \neq D_{M}^{\alpha}$ since $d^{\alpha}<1$. Note that in this simple example, the worlds $W^{\alpha}$ may be chosen independently of $\alpha$.

REMARK 4.17 Example 4.16 also shows that for $\alpha=\alpha_{0}$ every neighbourhood of $C^{\alpha_{0}}$ and hence every world $W^{\alpha}$ as in Theorem 4.12 may have non-void intersection with control sets different from $C^{\alpha_{0}}$.

\section{Relatively invariant $W$-control sets and invariance entropy}

In this section, we present an application of our results on relatively invariant $W$-control sets to invariance entropy.

We use the following version of invariance entropy for system (2.1). Let $K, Q$ be non-empty subsets of the state space $M$ where $K$ is a compact subset of $Q$, and assume that for every $x \in K$ there is a control $u \in \mathcal{U}$ such that $\varphi(t, x, u) \in$ int $Q$ for all $t>0$. Thus, in particular, $(K, Q)$ is an admissible pair in the sense of Kawan (2013, Definition 2.1).

For $\tau>0$, a set $\mathcal{S} \subset \mathcal{U}$ of control functions is called $\tau$-spanning for $(K, Q)$ if for every $x \in K$ there is $u \in \mathcal{S}$ with $\varphi(t, x, u) \in Q$ for all $t \in[0, \tau]$. By $r_{\text {inv }}(\tau, K, Q)$, we denote the minimal number of elements in such a set (if no finite $\tau$-spanning set exists $r_{\text {inv }}(\tau, K, Q):=\infty$ ).

Then the invariance entropy of $(K, Q)$ is defined by

$$
h_{\text {inv }}(K, Q):=\limsup _{\tau \rightarrow \infty} \frac{1}{\tau} \log r_{\text {inv }}(\tau, K, Q) \leqslant \infty
$$

If $K$ is contained in the interior of $Q$, the assumptions on $(K, Q)$ guarantee the existence of finite $\tau$-spanning sets.

Lemma 5.1 Fix a world $W$ and suppose for the compact set $Q:=\operatorname{cl} W$ that $W=\operatorname{int} Q$ and $W$ is connected.

(i) For every $W$-control set $D$ and compact subsets $K_{1}, K_{2} \subset D$ with non-void interior, one has

$$
h_{\text {inv }}\left(K_{1}, D\right)=h_{\text {inv }}\left(K_{2}, D\right) .
$$

(ii) Let $C_{i}, i \in\{1, \ldots, m\}$ be relatively invariant $W$-control sets and consider the union $\hat{K}:=\bigcup_{i=1}^{m} K_{i}$ of compact subsets $K_{i} \subset C_{i}$. Assume that local accessibility holds on $\hat{Q}:=\bigcup_{i=1}^{m} C_{i}$. Then

$$
h_{\text {inv }}(\hat{K}, Q)=h_{\text {inv }}(\hat{K}, \hat{Q})=\max _{i=1, \ldots, m} h_{\text {inv }}\left(K_{i}, C_{i}\right) .
$$

Proof. (i) Kawan (2013, Corollary 5.1) shows the assertion for control sets $D$ in $M$. The proof immediately applies also to $W$-control sets.

(ii) Observe that $\hat{K}$ is compact. Since $\hat{Q} \subset Q$, the inequality $h_{\text {inv }}(\hat{K}, Q) \leqslant h_{\text {inv }}(\hat{K}, \hat{Q})$ is trivial. For the converse, fix $\tau>0$ and consider a $\tau$-spanning set $\mathcal{S} \subset \mathcal{U}$ for $(\hat{K}, Q)$. Thus, for every $x \in \hat{K}$ there is a control $u \in \mathcal{S}$ with $\varphi(t, x, u) \in W=\operatorname{int} Q$ for all $t \in[0, \tau]$. For every $i$, relative invariance of $C_{i}$ with 
respect to $W$ implies that $\varphi(t, x, u) \in \operatorname{int} C_{i}$ for all $t \in[0, \tau]$ and all $x \in K_{i}$. Hence, $\mathcal{S}$ is also $\tau$-spanning for $(\hat{K}, \hat{Q})$. Taking the minimum over all $\tau$-spanning sets $\mathcal{S}$ for $(\hat{K}, Q)$ and then the limit for $\tau \rightarrow \infty$, one finds

$$
h_{\text {inv }}(\hat{K}, \hat{Q}) \leqslant h_{\text {inv }}(\hat{K}, Q) .
$$

The invariance entropy satisfies, cf. Kawan (2013, Proposition 2.7),

$$
h_{\text {inv }}(\hat{K}, \hat{Q})=\max _{i=1, \ldots, m} h_{\text {inv }}\left(K_{i}, \hat{Q}\right) .
$$

The proof is concluded by the observation that by relative invariance $h_{\text {inv }}\left(K_{i}, \hat{Q}\right)=h_{\text {inv }}\left(K_{i}, C_{i}\right)$ for every $i$.

The following theorem shows that for 'large' sets of initial values the invariance entropy is determined by the relatively invariant $W$-control sets. A major assumption will be that for every $x \in W$ there is a relatively invariant $W$-control set $C$ with $C \subset \mathrm{cl}_{W} \mathcal{O}^{W,+}(y)$ and there are only finitely many relatively invariant $W$-control sets. This property has been characterized in Theorem 4.4.

TheOREm 5.2 Consider control system (2.1). Fix a world $W$ and suppose for the compact set $Q:=\operatorname{cl} W$ that $W=\operatorname{int} Q$ and $W$ is connected. Let $K \subset Q$ be compact and assume that for every $x \in K$ there is a control $u \in \mathcal{U}$ such that $\varphi(t, x, u) \in \operatorname{int} Q$ for all $t>0$. Suppose that there are only finitely many relatively invariant $W$-control sets $C_{i}, i \in\{1, \ldots, n\}$ and that for every $x \in K$ there is $C_{i} \subset \operatorname{cl}_{W} \mathcal{O}^{W,+}(x)$. Finally, assume that the system is locally accessible on $\operatorname{cl} C_{i}$ for every $i$.

(i) For $i=1, \ldots, n$, let $K_{i}$ be a compact subset of $C_{i}$ with non-void interior. Then the invariance entropy of $(K, Q)$ satisfies

$$
h_{\text {inv }}(K, Q) \leqslant \max _{i=1,2, \ldots, n} h_{\text {inv }}\left(K_{i}, C_{i}\right) .
$$

(ii) Suppose that for every relatively invariant $W$-control set $C_{i}$ the intersection of $K$ with $C_{i}$ contains a compact subset $K_{i}$ with non-void interior. Then

$$
h_{\text {inv }}(K, Q)=\max _{i=1,2, \ldots, n} h_{\text {inv }}\left(K_{i}, C_{i}\right),
$$

where the maximum is taken over all relatively invariant $W$-control sets $C_{i}$.

Proof. Note that by Lemma 5.1(i), the invariance entropy $h_{\text {inv }}\left(K_{i}, C_{i}\right)$ is independent of the choice of the compact subset $K_{i} \subset C_{i}$ with non-void interior. First we show the following claim.

There are $\tau>0$, finitely many controls $w_{k} \in \mathcal{U}, k=1, \ldots, N$, and compact subsets $K_{i} \subset \operatorname{int} C_{i}$ with non-void interior such that for every $x \in K$ there is $w_{k}$ with $\varphi\left(t, x, w_{k}\right) \in W$ for all $t \in[0, \tau]$ and $\varphi\left(\tau, x, w_{k}\right) \in K_{i}$ for some $i$.

For every $x \in K$, there is $C_{i} \subset \operatorname{cl}_{W} \mathcal{O}^{W,+}(x)$ and hence int $C_{i} \subset \mathcal{O}^{W,+}(x)$. Choose compact sets $K_{i}^{\prime} \subset$ int $C_{i}$ with non-empty interior. Then for every $x \in K$, there are $\tau_{x} \geqslant 0$ and $u_{x} \in \mathcal{U}$ with $\varphi_{W}\left(\tau_{x}, x, u_{x}\right) \in \operatorname{int} K_{i}^{\prime}$ for some $i$. By compactness of $K$ and continuous dependence on initial values, there are finitely many $\tau_{j}>0$ and $u_{j} \in \mathcal{U}$ such that for every $x \in K$ there is $j$ with $\varphi\left(\tau_{j}, x, u_{j}\right) \in K_{i}^{\prime}$ for some $i$. Let $\tau_{\max }:=\max _{j} \tau_{j}$. For every $y \in K_{i}^{\prime}$, one finds a control $v_{y}$ and a neighbourhood $N(y)$ such that $\varphi\left(t, z, v_{y}\right) \in \operatorname{int} C_{i}$ for all $t \in\left[0, \tau_{\max }\right]$ and all $z \in N(y)$. Hence, compactness of $K_{i}^{\prime}$ implies that there are finitely many controls $v_{j}$ such that every $z \in K_{i}^{\prime}$ remains in int $C_{i}$ up to time $\tau_{\max }$.

Together, we have shown that there are $\tau:=\tau_{\max }>0$ and finitely many controls $w_{k}, k=1, \ldots, N$, such that for every $x \in K$ there is a control $w_{k}$ such that $\varphi\left(t, x, w_{k}\right) \in W$ for all $t \in[0, \tau]$ and $\varphi\left(\tau, x, w_{k}\right) \in$ 
int $C_{i}$ for some $i$. By finiteness of the number of controls and compactness of $K$, it follows that there are compact subsets $K_{i} \subset$ int $C_{i}$ with non-void interior such that for every $x \in K$ there is $w_{k}$ with $\varphi\left(\tau, x, w_{k}\right) \in$ $K_{i}$ for some $i$. This proves the claim.

(i) In view of Lemma 5.1(ii), it suffices to show that

$$
h_{\text {inv }}(K, Q) \leqslant h_{\text {inv }}(\hat{K}, Q) \text {, }
$$

where $\hat{K}:=\bigcup_{i=1}^{m} K_{i}$ with compact sets $K_{i}$ constructed in the claim.

Let $\mathcal{S} \subset \mathcal{U}$ be a $\tau^{\prime}$-spanning set for $(\hat{K}, Q)$ with minimal cardinality $r_{\text {inv }}(\tau, \hat{K}, Q)$. The claim shows that there are $\tau>0$, finitely many controls $w_{k} \in \mathcal{U}, k=1, \ldots, N$, such that for every $x \in K$ there is $w_{k}$ with $\varphi\left(t, x, w_{k}\right) \in W$ for all $t \in[0, \tau)$ and $\varphi\left(\tau, x, w_{k}\right) \in K_{i}$ for some $i$. Then the concatenations of the controls in $\mathcal{S}$ with the $N$ controls $w_{k}$ are $\left(\tau+\tau^{\prime}\right)$-spanning for $(K, Q)$. Hence, their number $N \cdot r_{\text {inv }}(\tau, \hat{K}, Q)$ is an upper bound for $r_{\text {inv }}\left(\tau+\tau^{\prime}, K, Q\right)$. Since $N$ is independent of $\tau^{\prime}$, it follows that

$$
h_{\text {inv }}(K, Q)=\limsup _{\tau^{\prime} \rightarrow \infty} \frac{1}{\tau^{\prime}} \log r_{\text {inv }}\left(\tau^{\prime}, K, Q\right) \leqslant h_{\text {inv }}(\hat{K}, Q) .
$$

(ii) Let $K_{i} \subset K \cap C_{i}$ be compact with non-void interior. By relative invariance of $C_{i}$, one has

$$
h_{\text {inv }}\left(K_{i}, C_{i}\right)=h_{\text {inv }}\left(K_{i}, Q\right) \leqslant h_{\text {inv }}(K, Q) \text {. }
$$

Assertion (i) shows that also the converse inequality for the maximum holds.

REMARK 5.3 For the invariance entropy of control sets, Kawan (2012, 2013, Chapter 5) has proved good upper bounds of the invariance entropy $h_{\text {inv }}(K, D)$ for compact subsets $K \subset D$ with non-void interior. These estimates are given in terms of Floquet exponents for regular periodic solutions in the interior of the control set. The proofs remain valid for $W$-control sets. Thus, Theorem 5.2 will allow us to prove similar estimates for more general situations by reducing them to estimates for $W$-control sets, cf. Section 6 for an example.

\section{Invariance entropy for a tank reactor}

In this section, we illustrate Theorem 5.2 by an application to control of a continuous stirred tank reactor with Arrhenius' dynamics, cf., e.g. Poore (1974) or Golubitsky \& Schaeffer (1985). We will briefly recall the results on the controllability structure given in Colonius \& Kliemann (2000, Section 9.1) which are partially based on numerical evidence, and discuss $W$-invariant control sets and an application of Theorem 5.2 for invariance entropy.

Consider the following model of a continuous stirred tank reactor given by

$$
\begin{aligned}
\left(\begin{array}{c}
\dot{x}_{1} \\
\dot{x}_{2}
\end{array}\right) & =\left(\begin{array}{c}
-x_{1}-a\left(x_{1}-x_{c}\right)+B \alpha\left(1-x_{2}\right) e^{x_{1}} \\
-x_{2}+\alpha\left(1-x_{2}\right) e^{x_{1}}
\end{array}\right)+u(t)\left(\begin{array}{c}
x_{c}-x_{1} \\
0
\end{array}\right) \\
& =X_{0}(x)+u(t) X_{1}(x) .
\end{aligned}
$$

Here $x_{1}$ is the (dimensionless) temperature; $x_{2}$ is the product concentration; and $a, \alpha, B$ and $x_{c}$ are positive constants. The parameter $x_{c}$ is the coolant temperature, and hence the control affects the heat 
transfer coefficient. Specifically, we take

$$
a=0.15, \alpha=0.05, B=7.0, x_{c}=1.0, U^{\rho}=[-\rho, \rho] \quad \text { with } 0<\rho \leqslant 0.15 .
$$

Because of the physical constraints, we consider the system in the set $[0, \infty) \times[0,1] \subset \mathbb{R}^{2}$. For each fixed $u \in U$, equation (6.1) has three fixed points. Let $y_{i}=\alpha e^{z_{i}} /\left(1+\alpha z_{i}\right), i=0,1,2$, where $z_{1}<z_{0}<z_{2}$ are the zeros of the transcendental equation

$$
-z-(a+u)\left(z-x_{c}\right)+B \alpha\left[1-\frac{\alpha e^{z}}{1+\alpha e^{z}}\right] e^{z}=0 .
$$

Then these fixed points are given as two stable ones $x_{1}=\left(z_{1}, y_{1}\right)$ and $x_{2}=\left(z_{2}, y_{2}\right)$ and a hyperbolic one $x_{0}=\left(z_{0}, y_{0}\right)$, i.e. the linearization about $x_{0}$ has one negative and one positive eigenvalue. The phase portrait of the uncontrolled equation is indicated in Fig. 1.

The system satisfies the Lie algebra rank condition (ARC) at every point of the forward invariant set $(0, \infty) \times(0,1)$. The numerical computations indicate that for the parameter values (6.2) the set $M=$ $[0,7] \times[0,1]$ contains exactly three control sets $C_{1}^{\rho}, C_{2}^{\rho}$ and $D^{\rho}$ containing the fixed points $x_{i}(u), i=$ $1,2,0$, for $u \in \operatorname{int} U^{\rho}=(-\rho, \rho)$ in their interior. The control sets $C_{1}^{\rho}$ and $C_{2}^{\rho}$ are invariant; the control set $D^{\rho}$ is variant, see Fig. 1 for the situation with $\rho=0.15$.

An interesting feature of this system is that the stable fixed point $x_{2}$ with the highest product concentration cannot be realized for technical reasons, hence it is of interest to keep the system near the hyperbolic equilibrium $x_{0}$.

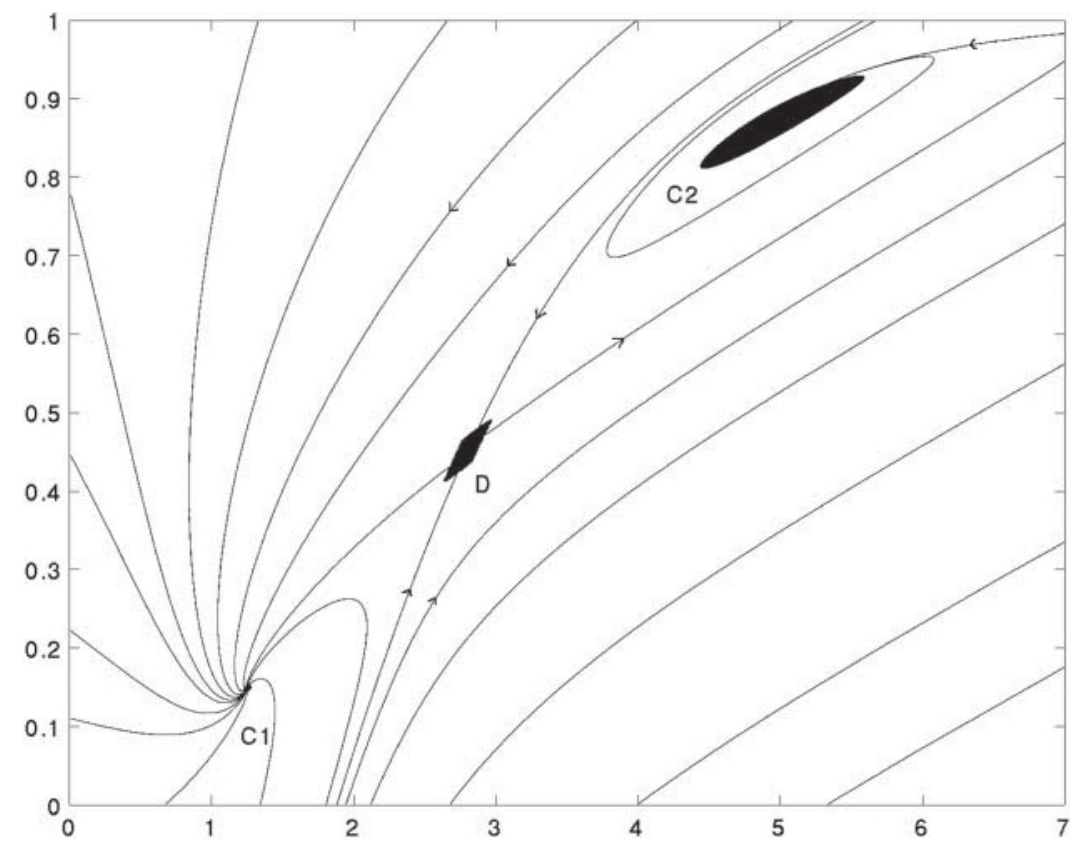

FIG. 1. Phase portrait of the continuous flow stirred tank reactor and control sets. 
The following result (given here in the notation from the present paper) is a consequence of Colonius $\&$ Kliemann (2000, Theorem 9.1.1). We denote the reachable set from $y \in M$ with controls in $\mathcal{U}^{\rho}$ by $\mathcal{O}^{\rho,+}(y)$.

Theorem 6.1 Consider system (6.1) and (6.2) with control range $U^{\rho}=[-\rho, \rho], 0<\rho \leqslant 0.15$. Denote by $M^{+}(u)$ and $M^{-}(u)$ the stable and the unstable manifolds, respectively, in $M$ of the hyperbolic fixed point $x_{0}(u), u \in[-0.15,0.15]$. Then the control set $D^{\rho}$ is given by

$$
\operatorname{int} D^{\rho}=\operatorname{int} \bigcup_{u_{1}, u_{2} \in U^{\rho}}\left(M^{+}\left(u_{1}\right) \cap M^{-}\left(u_{2}\right)\right) \text {, }
$$

and the domain of attraction of $D^{\rho}$ defined as $\mathbf{A}\left(D^{\rho}\right):=\left\{y \in M \mid \operatorname{cl} \mathcal{O}^{\rho,+}(y) \cap D^{\rho} \neq \emptyset\right\}$ satisfies

$$
\mathbf{A}\left(D^{\rho}\right)=\operatorname{int} \bigcup_{u \in U^{\rho}} W^{+}(u)
$$

The boundary of $\mathbf{A}\left(D^{\rho}\right)$ consists of the stable manifolds corresponding to $u_{1}=-\rho$ and $u_{2}=\rho$.

Figure 2 shows the set $\mathbf{A}\left(D^{0.15}\right)$.

We will apply Theorem 5.2 to the system with controls in $\mathcal{U}^{\rho_{0}}$ which have range in $U^{\rho_{0}}=\left[-\rho_{0}, \rho_{0}\right]$ and worlds $W^{\rho}:=\mathbf{A}\left(D^{\rho}\right), \rho \in\left(0, \rho_{0}\right)$. The sets $W^{\rho}$ are open, relatively compact and connected. For every $\rho \in\left(0, \rho_{0}\right)$, the control set $D^{\rho}$ of the $\rho$-system is contained in a relatively invariant $W^{\rho}$-control set $C^{\rho_{0}}\left(W^{\rho}\right)$ of the system with controls in $\mathcal{U}^{\rho_{0}}$ : In fact, an analysis of the phase portraits near the hyperbolic equilibria of the system with constant controls reveals that the sets $D^{\rho}$ can be left by trajectories in

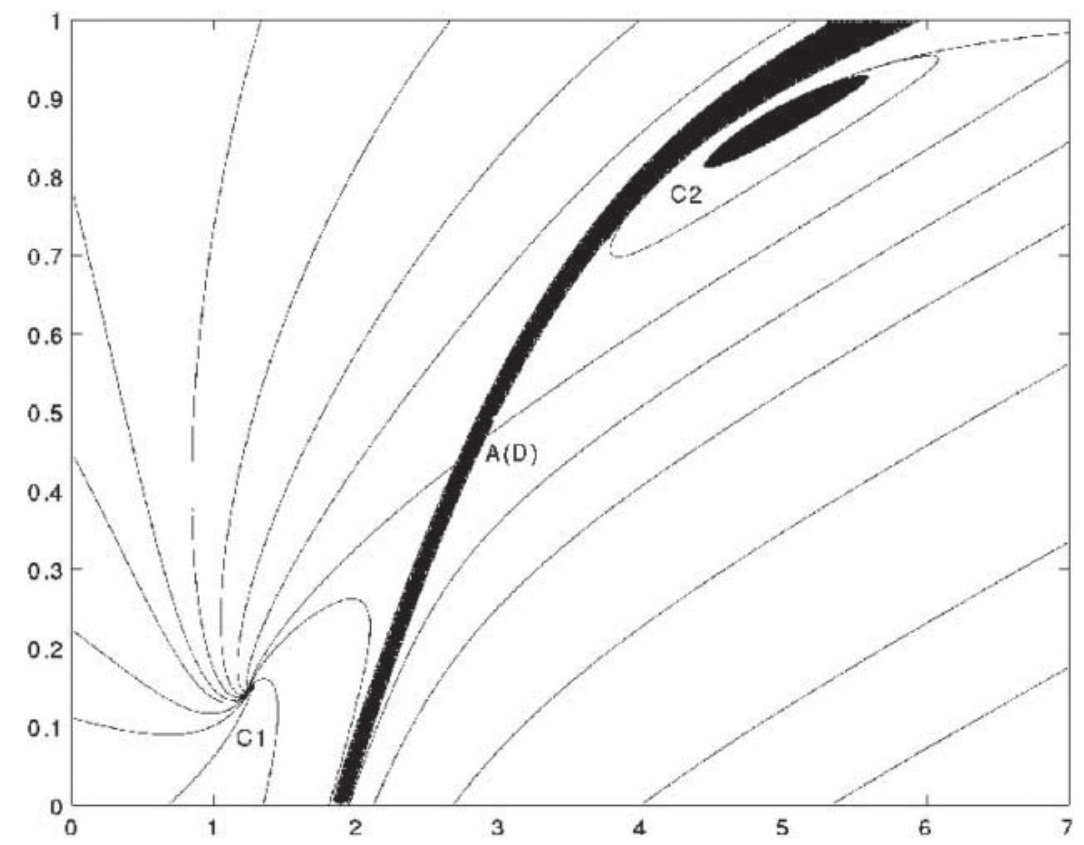

FIG. 2. World $W^{0.15}$ of the continuous flow stirred tank reactor. 
$W^{\rho}$ with controls $u \in\left[-\rho_{0},-\rho\right) \cup\left(\rho, \rho_{0}\right]$. Choose any initial point $x_{0} \in \operatorname{int} D^{\rho}$. Let $\mathcal{O}^{\rho_{0}, W^{\rho},+}\left(x_{0}\right)$ be the reachable set within $W^{\rho}$ with controls in $\mathcal{U}^{\rho_{0}}$. Every point in this set can be steered back to $D^{\rho}$ without leaving $W^{\rho}$. It follows that

$$
C^{\rho_{0}}\left(W^{\rho}\right):=D^{\rho} \cup \mathcal{O}^{\rho_{0}, W^{\rho},+}\left(x_{0}\right)
$$

is a relatively invariant $W^{\rho}$-control set for the system with controls in $\mathcal{U}^{\rho_{0}}$.

Theorem 5.2 implies that for every compact set $K \subset W^{\rho}$ intersecting the relatively invariant $W^{\rho}$ control set $C^{\rho_{0}}\left(W^{\rho}\right)$ in a set with non-void interior the invariance entropy coincides with the invariance entropy for $C^{\rho_{0}}\left(W^{\rho}\right)$. Now the results in Kawan (2013, Chapter 5) can be applied for $\rho_{0}>0$, small enough, to yield that the invariance entropy of $C^{\rho_{0}}\left(W^{\rho}\right)$ satisfies for every compact subset $K \subset C^{\rho_{0}}\left(W^{\rho}\right)$ the following estimate: Let $\lambda(u, x)$ be the positive Floquet exponent of any $T$-periodic trajectory $\varphi(\cdot, x, u)$ corresponding to a $T$-periodic control $u \in \mathcal{U}^{\rho_{0}}, T>0$, with $(x, u) \in \operatorname{int} C^{\rho_{0}}\left(W^{\rho}\right) \times\left(-\rho_{0}, \rho_{0}\right)$. Then

$$
h_{\text {inv }}^{\rho_{0}}\left(K, C^{\rho_{0}}\left(W^{\rho}\right)\right) \leqslant \lambda(u, x) .
$$

In fact, Kawan (2013, Propositions 5.9 and 5.12) shows that for control-affine systems the invariance entropy can be bounded above by the sum of the positive Floquet exponents, provided that uniform hyperbolicity holds. Uniform hyperbolicity of the control system restricted to $C^{\rho_{0}}\left(W^{\rho}\right)$ (more precisely, its corresponding control flow) can be verified, since the equilibrium of the uncontrolled system is hyperbolic. Then roughness of the Sacker-Sell spectrum (or the Morse spectrum, cf., e.g. Colonius \& Kliemann (2000, Corollary 5.3.11)) implies that uniform hyperbolicity also holds for $\rho_{0}>0$ small enough.

\section{Conclusions}

This paper has studied $W$-control sets which are defined as maximal subsets of complete approximate controllability within a safe region or world $W$ in the state space $M$. These generalizations of control sets (which are obtained in the special case $W=M$ ) share many properties with control sets which are of some interest for the analysis of the global behaviour of control systems and random dynamical systems.

We have also given an application to the recently introduced notion of invariance entropy (or topological feedback entropy). For sufficiently large sets of initial values, the relatively invariant $W$-control sets determine the invariance entropy. This is of particular interest, since for control sets $D$ in $M$ good upper estimates for the invariance entropy are available, cf. Remark 5.3 and the example presented in Section 6.

The fact that invariance entropy is determined by the behaviour of the system on certain subsets of the state space is reminiscent of the classical fact that the topological entropy of dynamical systems is determined by the restriction to the non-wandering set; cf. Katok (2007). Hence, despite many differences, this notion of invariance entropy shares another property with topological entropy.

\section{Acknowledgements}

We thank the anonymous reviewers for the careful reading and the constructive criticism which led to an improvement of the paper.

\section{Funding}

F.C. was supported by DFG grant Co 124/19-1. 


\section{REFERENCES}

Arnold, L. \& Kliemann, W. (1987) On unique ergodicity for degenerate diffusions. Stochastics, 21, 41-61. Aubin, J.-P. \& Frankowska, H. (1990) Set-Valued Analysis. Boston: Birkhäuser.

Berger, M. \& Gostiaux, B. (1988) Differential Geometry: Manifolds, Curves, and Surfaces. Graduate Text in Mathematics, vol. 115. Berlin: Springer.

Colonius, F., Gayer, T. \& Kliemann, W. (2008) Near invariance for Markov diffusions. SIAM J. Appl. Dyn. Syst., 7, 79-107.

Colonius, F. \& Kliemann, W. (2000) The Dynamics of Control. Boston: Birkhäuser.

Crouch, P. Lecture Notes on Geometric Non Linear Systems Theory. Control Theory Centre Report- University of Warwick, vol. 99. Warwick: University of Warwick Control Theory Center (without year specification).

Demers, M. F. \& Young, L.-S. (2006) Escape rates and conditionally invariant measures. Nonlinearity, 19, $377-397$.

Gayer, T. (2004) Control sets and their boundaries under parameter variation. J. Differential Equations, 201, $177-200$.

Golubitsky, M. \& SchaefFer, D. (1985) Singularities and Groups in Bifurcation Theory. Berlin: Springer.

Graf, I. (2011) Boundaries of local no-return sets for control systems. J. Dyn. Control Systems, 17, 311-328.

Kаток, А. (2007) Fifty years of entropy in dynamics: 1958-2007. J. Mod. Dyn., 1, 545-596.

KaWAN, C. (2012) Invariance entropy of control sets. SIAM J. Control Optim., 49, 732-751.

Kawan, C. (2013) Invariance Entropy of Deterministic Control Systems. An Introduction. Lecture Notes Mathematics, vol. 2089. Berlin: Springer.

LeE, J. M. (2013) Introduction to Smooth Manifolds, 2nd edn. Graduate Texts in Mathematics, vol. 218. Berlin: Springer.

Nair, G., Evans, R. J., Mareels, I. \& Moran, W. (2004) Topological feedback entropy and nonlinear stabilization. IEEE Trans. Automat. Control, 49, 1585-1597.

Patrão, M. \& San Martin, L. A. B. (2007) Morse decomposition of semiflows on fiber bundles. Discrete Contin. Dyn. Syst., 17, 113-139.

Poore, A. B. (1974) A model equation arising from chemical reactor theory. Arch. Ration. Mech. Anal., 52, 358-388.

Sontag, E. (1998) Mathematical Control Theory. Berlin: Springer.

Tomlin, C., PAPPAS, G. J. \& SASTRY, S. S. (1998) Conflict resolution for air traffic management: a study in multiagent hybrid systems. IEEE Trans. Automat. Control, 43, 509-521. 